Illustrations dans différents domaines 
Collection SFN 10 (2010) 507-528

(C) Owned by the authors, published by EDP Sciences, 2010

DOI: $10.1051 / \mathrm{sfn} / 2010011$

\title{
Phonons et vibrations dans les fullerènes, les nanotubes de carbone et leurs composés
}

\author{
J. Cambedouzou ${ }^{1}$ et S. Rols ${ }^{2}$ \\ ${ }^{1}$ Laboratoire de Physique des Solides, UMR CNRS 8502, Université Paris-Sud 11, \\ 91405 Orsay Cedex, France \\ 2 Institut Laue Langevin, 6 rue Jules Horowitz, 38042 Grenoble Cedex 9, France
}

\begin{abstract}
Résumé. À travers quelques résultats expérimentaux marquants, nous illustrons l'apport des techniques de diffusion inélastique des neutrons à la compréhension des dynamiques de vibration et de réseau des fullerènes et des nanotubes de carbone. L'accent est particulièrement mis sur l'étude des excitations intra et inter-moléculaires des fullerènes de $\mathrm{C}_{60}$, ainsi que sur la transition dynamique ordre/désordre caractéristique de ces molécules. En outre, une part significative de ce chapitre est dédiée à divers composés d'intercalation et d'insertion des fullerènes et des nanotubes de carbone, comme le co-cristal "fullerène-cubane" formé d'un arrangement de molécules de formes sphérique et cubique, ou encore les composés appelés "peapods", dans lesquels des fullerènes $\mathrm{C}_{60}$ sont insérés à l'intérieur de nanotubes de carbone monofeuillets.
\end{abstract}

\section{INTRODUCTION}

\subsection{Quelques repères historiques}

Jusqu'en 1985, le carbone n'était essentiellement connu que sous deux formes allotropiques distinctes : le graphite et le diamant (voir la figure 1). Ces matériaux, bien que tous deux exclusivement constitués de carbone, n'en possèdent pas moins des propriétés physiques bien distinctes. Par exemple, le graphite est noir et friable (il est utilisé pour les mines de crayon à papier) alors que le diamant est transparent et fait figure de référence pour sa dureté. Ces propriétés physiques parfois opposées, pour des matériaux composés du même type d'atome, reflètent des structures cristallines différentes. Le graphite est constitué de plans dans lesquels les atomes de carbone sont fortement liés par des interactions covalentes, mais ces plans sont eux-mêmes plus faiblement liés par des interactions de van der Waals. Il s'ensuit une grande friabilité du matériau à l'échelle macroscopique. Dans le cas du diamant, les atomes de carbone sont liés par des interactions covalentes fortes (hybridation de type sp3) de telle manière que chaque atome de carbone se trouve au centre d'un tétraèdre d'atomes de carbone. À l'échelle macroscopique, une telle structure se traduit par une grande dureté.

Lors d'une expérience qui visait à la synthèse de molécules dans des conditions proches de celles régnant dans le milieu inter-stellaire, H. Kroto, R.F. Curl et R.E. Smalley [1] mirent en évidence une nouvelle molécule exclusivement constituée de carbone : le fullerène $\mathrm{C}_{60}$ qui peut être considéré comme une troisième forme allotropique du carbone.

Les fullerènes $\mathrm{C}_{60}$ sont des molécules singulières car elles conservent une très haute symétrie malgré le nombre important d'atomes qui les composent. On peut décrire la structure d'un $\mathrm{C}_{60}$ comme l'assemblage de 60 atomes de carbone répartis aux sommets d'un icosaèdre tronqué (voir la figure 2). Un $\mathrm{C}_{60}$ présente donc 32 faces se répartissant en 12 pentagones et 20 hexagones, exactement comme

This is an Open Access article distributed under the terms of the Creative Commons Attribution-Noncommercial License 3.0, which permits unrestricted use, distribution, and reproduction in any noncommercial medium, provided the original work is properly cited. 


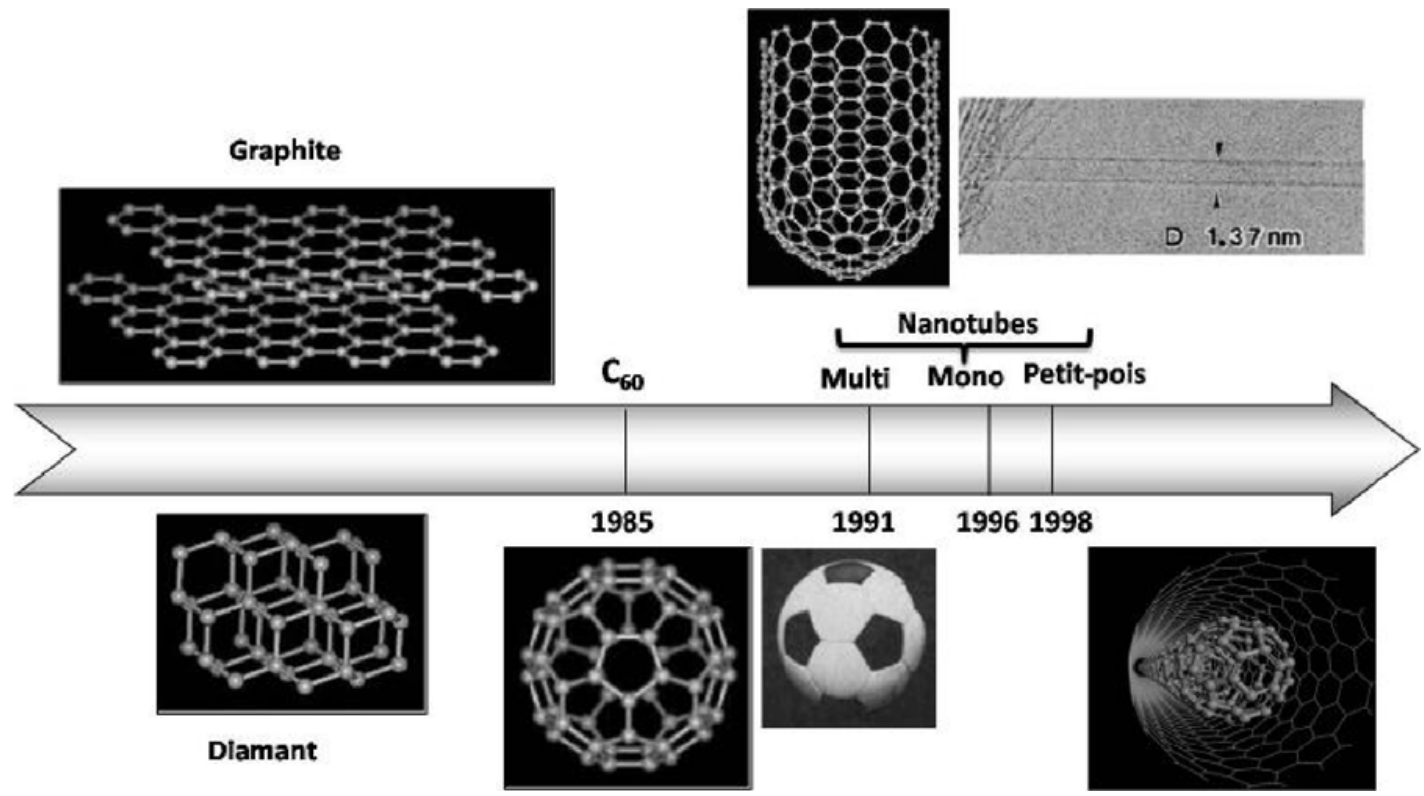

Figure 1. Évolution de la famille des molécules de carbone au cours de l'histoire.

un ballon de football "classique" 1 ", ainsi que 90 arêtes. Cette structure particulière a inspiré le nom de la molécule, qui a été baptisée fullerène en hommage à l'architecte Richard Buckminster Fuller, concepteur du dôme géodésique édifié sur le pavillon des Etats-Unis lors de l'exposition universelle de Montréal en 1967.

D'autres structures moléculaires en cage de carbone furent synthétisées et portent aussi le nom de fullerènes. On trouve parmi celles-ci le fullerène $C_{70}$ à la forme ovoïde, le fullerène $C_{84}$, etc... Afin d'étudier et de comprendre les propriétés physiques de ces nouvelles molécules, des tentatives de synthèses en grande quantité de $\mathrm{C}_{60}$ furent menées. C'est au cours de l'une d'entre elles, en utilisant la méthode de l'arc électrique et en introduisant un catalyseur, que furent identifiés pour la première fois $^{2}$ des nanotubes de carbone par S. Iijima en 1991 [2].

\subsection{Intérêt de ce cours-exemple}

Les fullerènes sont souvent utilisés pour illustrer des notions de physique liées aux cristaux moléculaires. En effet, leur très haute symétrie, ainsi que leur relative simplicité (un seul type d'atome - le carbone par molécule) rendent les calculs simples et procurent ainsi aux physiciens des systèmes pédagogiques, dont le plus étudié et le plus utilisé est le fameux fullerène $\mathrm{C}_{60}$. De plus, ces composés possèdent un diagramme de phase riche, où des composés polymères peuvent être synthétisés dans des conditions particulières de pression, de température et/ou de dopage (voir diagramme de phase en Fig. 3). Ces phases polymères sont formées d'un arrangement des cages liées par des ponts covalents. En fonction du nombre de liaisons covalentes par molécule, les cages subissent des déformations et leur structure supramoléculaire change. Le cristal moléculaire se rigidifie et prend alors progressivement un caractère de cristal atomique.

\footnotetext{
${ }^{1}$ Les ballons de type "Teamgeist" apparus en 2006 ont remplacé ces ballons dans les compétitions officielles. Ces nouveaux ballons ne comptent que 14 faces.

2 Oberlin et al. (J. Cryst. Growth 32(3), 335 (1976)) avaient en fait observé des nanotubes de carbone 15 ans auparavant, mais sans écho de la part de la communauté scientifique.
} 
Ces phases ont été présentées dans le chapitre 1 de cet ouvrage et ne seront pas rediscutées dans ce cours. Nous nous proposons ici de discuter la dynamique vibrationnelle du fullerène $\mathrm{C}_{60}$ en tant que cristal moléculaire à 3 dimensions. Nous montrerons ensuite l'effet de l'insertion de molécules invitées dans les sites octaédriques du réseau cubique sur la dynamique ainsi que sur le diagramme de phase. Nous discuterons ensuite la dynamique des nanotubes de carbone monofeuillets organisés en faisceaux. Pour finir, nous présenterons les résultats de l'étude de la dynamique de molécules issues de l'association parfaite des $\mathrm{C}_{60}$ et des nanotubes monofeuillets : les nano-petits-pois ou "peapods", formés de chaînes $1 \mathrm{D}$ de $\mathrm{C}_{60}$ confinées dans des nanotubes. Nous évoluerons ainsi d'un cristal moléculaire 3D vers un cristal moléculaire 1D confiné.

\section{LES VIBRATIONS DANS UN CRISTAL MOLÉCULAIRE TRIDIMENSIONNEL : LE FULLERÈNE C $_{60}$ EN PHASE SOLIDE ET SES COMPOSÉS D'INSERTION}

\subsection{Le $\mathrm{C}_{60}$ cristallin pur}

\subsubsection{Structure, Symétrie et "transition ordre-désordre"}

La symétrie icosaèdrique du $\mathrm{C}_{60}$ impose la présence de différents éléments de symétrie. Au niveau des axes de symétrie, on dénombre 6 axes d'ordre 5 qui relient les centres de deux pentagones opposés, 10 axes d'ordre 3 qui relient les centres de deux hexagones opposés, et enfin 15 axes d'ordre 2 qui relient les milieux de deux doubles liaisons opposées. Un exemple de chacun de ces axes de symétrie est donné sur la figure 2 .
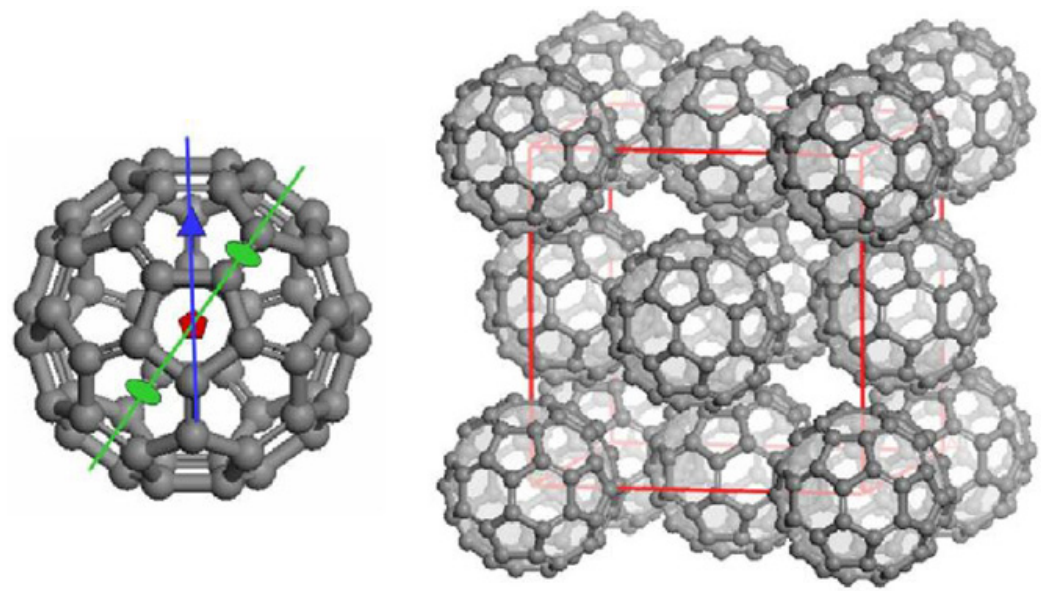

Figure 2. Gauche : Schéma d'un fullerène $\mathrm{C}_{60}$ avec un axe d'ordre 5 (pentagone), un axe d'ordre 3 (triangle) et un axe d'ordre 2 (ovale). - Droite: Structure cristalline du fullerène $\mathrm{C}_{60}$.

Enfin, deux types de liaisons chimiques assurent la cohésion de la molécule : on trouve 60 liaisons simples communes à un hexagone et un pentagone adjacents, et 30 doubles liaisons communes à 2 hexagones adjacents.

Dans sa phase cristalline, les $\mathrm{C}_{60}$ s'organisent sous la forme d'un cristal moléculaire dans lequel les molécules interagissent entre elles par des liaisons faibles de type van der Waals. Soumis à certains traitements à haute pression et haute température (HPHT), elles peuvent aussi former des liaisons covalentes entre elles et aboutir à des réseaux inter-connectés comme nous l'avons discuté en introduction ainsi que dans le chapitre 1. La figure 3 montre un diagramme de phase publié par Davydov en 2000 [3]. Nous renvoyons le lecteur intéressé à la signification des différents domaines à 


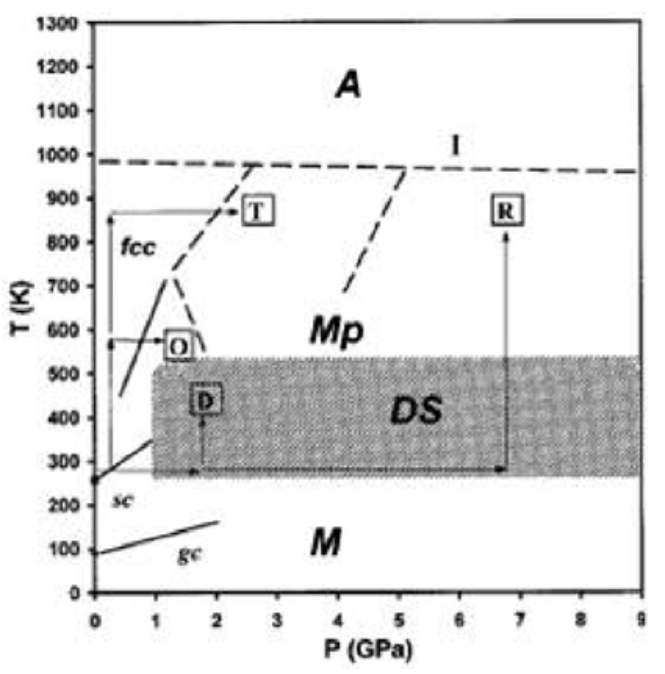

Figure 3. Diagramme de phase en pression et température des fullerènes $\mathrm{C}_{60}$. D'après la référence [3]

l'article original. Nous focaliserons dans ce chapitre notre attention aux domaines notés fcc, sc et gc qui renvoient au domaines ou la structure du réseau des $\mathrm{C}_{60}$ est respectivement "cubique face centré", "cubique simple" et "vitreuse".

À température et pression ambiantes, les molécules de $\mathrm{C}_{60}$ se trouvent dans une phase de cristal plastique, dans lequel ils sont animés d'un mouvement de rotation isotrope quasi-libre. Cette structure peut être décrite en utilisant le groupe d'espace $\mathrm{Fm} 3 \mathrm{~m}$, relatif à un réseau cubique faces centrées (fcc) de paramètre de maille $14.15 \AA$, où le motif correspond à une molécule de $\mathrm{C}_{60}$ présentant une désorientation complète - i.e. les molécules de $\mathrm{C}_{60}$ sont assimilables à des sphères homogènes. Ces mouvements de réorientations rapides quasi-isotropes dans la phase désordonnée (T > $260 \mathrm{~K})$ ont été mis en évidence par des techniques expérimentales telles que la résonance magnétique nucléaire [6], la diffusion diffuse des rayons $\mathrm{X}$ [7] et la diffusion quasi-élastique des neutrons [8]. Si l'on abaisse la température en dessous de $\mathrm{T}_{c} \simeq 260 \mathrm{~K}$, le cristal de $\mathrm{C}_{60}$ subit une transition dynamique vers une phase dite "ordonnée", dans laquelle les $\mathrm{C}_{60}$ restent sur les mêmes sites cristallographiques que dans la phase désordonnée, mais ne présentent plus de mouvement de rotation. Leur diffusion rotationnelle est bloquée, et la mise en ordre de leur orientation fait que la symétrie de la maille est abaissée. La structure ordonnée peut être décrite dans le groupe d'espace $\mathrm{Pa} \overline{3}$. Cependant, les orientations relatives entre molécules adjacentes peuvent encore fluctuer entre deux orientations distinctes notées $\mathrm{H}$ et $\mathrm{P}$ (voir figure 4) dont les populations respectives dépendent de la température. Pour des températures inférieures à $90 \mathrm{~K}$, il se produit un gel orientationnel au dessous duquel les molécules $\mathrm{C}_{60}$ restent figées dans leurs orientations. Le $\mathrm{C}_{60}$ se trouve alors dans un état de "verre orientationnel". Nous appellerons dans la suite "phase ordonnée" la phase cubique simple $(\mathrm{T}<260 \mathrm{~K})$, étant bien entendu qu'une certaine quantité de désordre orientationnel y persiste.

\subsubsection{Dynamique vibrationnelle dans la phase ordonnée $(T<260 \mathrm{~K})$}

La caractéristique majeure d'un cristal moléculaire est la très forte différence entre les valeurs importantes des constantes de forces intramoléculaires (i.e. caractéristiques des liaisons - covalentes - entre atomes de la même molécule) et intermoléculaires, plus souples. Ceci entraîne un découplage des mouvements des atomes de carbone à la surface d'une molécule par rapport aux mouvements d'ensemble (rotation, translation du centre de masse) de cette molécule. Dans le cas du $\mathrm{C}_{60}$ il s'ensuit une très nette séparation entre les bandes de fréquences à basse énergie $(\mathrm{E}<10 \mathrm{meV})$ caractéristiques 

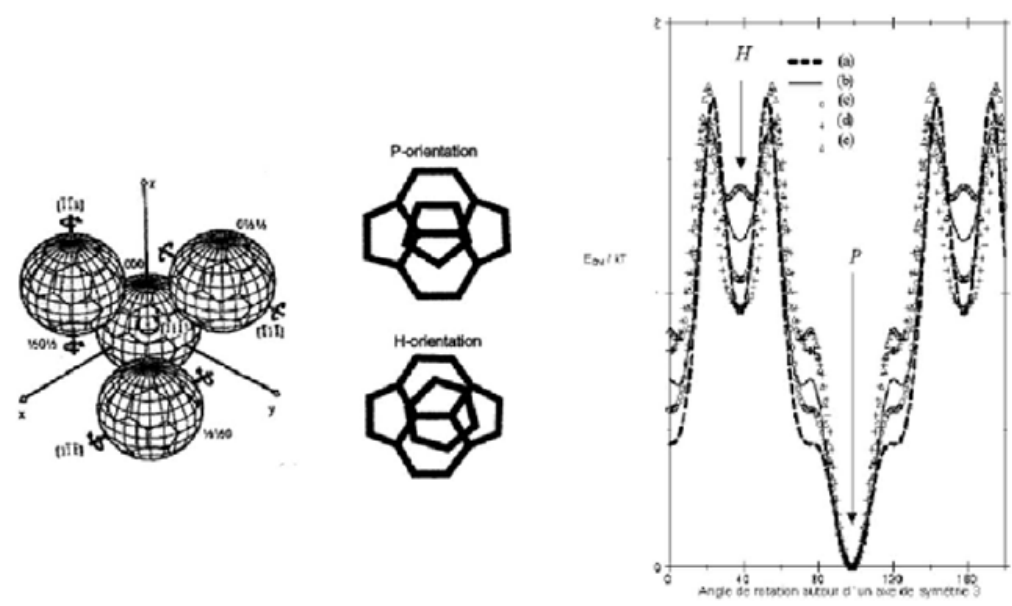

Figure 4. Gauche : Représentation schématique de l'orientation des fullerènes $\mathrm{C}_{60}$ dans leur phase ordonnée de groupe d'espace $\mathrm{Pa} \overline{3}-$ Milieu : Représentation de l'orientation d'un $\mathrm{C}_{60}$ par rapport à un pentagone ou un hexagone de la molécule voisine suivant que son orientation est "P" ou "H". D'après la référence [4] - Droite : Energie potentielle de rotation en fonction de l'angle de rotation autour d'un axe d'ordre 3. D'après la référence [5].

des modes vibrationnels dits "de réseau", et celles des modes de vibrations dits "moléculaires", de plus haute énergie ( $\mathrm{E}>25 \mathrm{meV}$ ). Alors que les modes de réseau (phonons, librations) n'impliquent pas de déformation de la cage du $\mathrm{C}_{60}$, les modes de vibrations moléculaires impliquent eux des déformations périodiques de la structure de cette dernière. Ces différents domaines d'énergie sont séparés par une bande où n'apparaît aucun mode, généralement appelée gap, et qui s'étend sur un domaine d'énergie large de plus de $20 \mathrm{meV}$.

\section{Les vibrations moléculaires}

Une molécule de $\mathrm{C}_{60}$ possède 174 modes de vibrations moléculaires. La symétrie icosaèdrique, de très grande symétrie, impose qu'un grand nombre de ces modes sont dégénérés (voir cours de F. Guillaume et M. Couzi sur la symétrie des modes de vibrations). Au final, seules 46 fréquences distinctes pourront être mesurées ${ }^{3}$. La décomposition des modes en représentation irréductible s'écrit :

$$
2 A_{g}+A_{u}+3 T_{g}^{1}+4 T_{u}^{1}+4 T_{g}^{2}+5 T_{u}^{2}+6 G_{g}+6 G_{u}+8 H_{g}+7 H_{u}
$$

Parmi ces 46 modes, seuls 4 sont actifs en absorption infrarouge, et 10 sont actifs en spectroscopie Raman. Les 32 autres modes de vibrations ne peuvent alors être étudiés qu'avec la diffusion inélastique des neutrons (DIN).

Les premières mesures de DIN ont été réalisées sur des poudres de $\mathrm{C}_{60}$, donnant ainsi accès à la densité d'état généralisée (GDOS) des modes de vibrations. La première détermination expérimentale de la GDOS d'une poudre de fullerènes $\mathrm{C}_{60}$ a été réalisée par Capelletti et al. au NIST (Washington) [10] sur un spectromètre à filtre-analyseur (voir cours de B. Hennion). Elles ont été suivies par des mesures de bien meilleure qualité l'année suivante à ISIS sur le spectromètre à temps de vol - filtre analyseur TFXA (Oxford) par Coulombeau et al. [11] ${ }^{4}$. La figure 5 (droite) présente la GDOS mesurée sur une poudre de $\mathrm{C}_{60}$ et réalisée à l'ILL sur les spectromètres $\mathrm{IN} 4 \mathrm{C}$ et $\mathrm{IN} 1 \mathrm{BeF}$. IN4C est un spectromètre à temps de vol installé sur une source thermique (voir cours de J. Ollivier et J.-M. Zanotti), alors qu'IN1BeF est un spectromètre à filtre analyseur installé sur une source chaude. Ces deux spectromètres ont leurs

\footnotetext{
3 Nous appellerons souvent un "mode" l'ensemble des modes dégénérés de même fréquence.

4 Des mesures de moins bonne qualité avaient néanmoins été rapportées plus tôt (1991) par K. Prassides [12] sur le même appareil.
} 

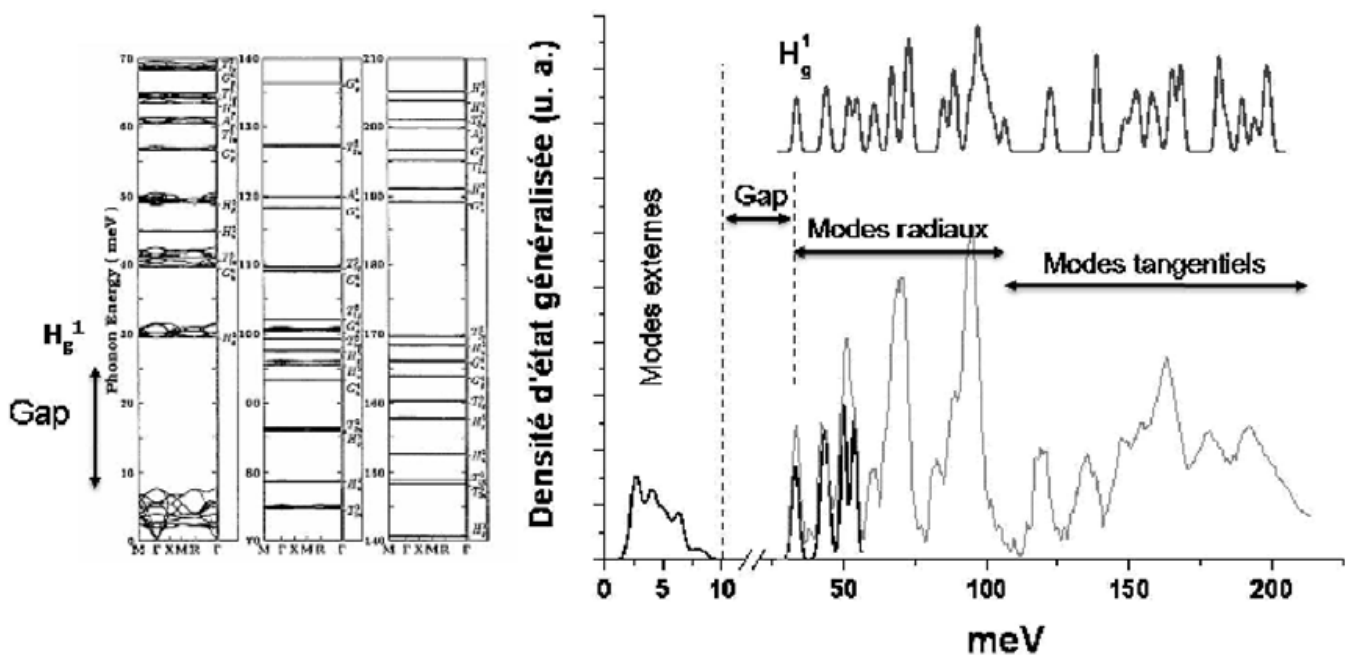

Figure 5. Gauche : Courbes de dispersion calculées par dynamique des réseaux d'un cristal de fullerènes $\mathrm{C}_{60}$ (tiré de la référence [9]) - Droite : Densité d'états de phonons d'un cristal de fullerènes $\mathrm{C}_{60}$ mesurée sur les spectromètres IN4C (noir) et IN1BeF (gris clair) à l'ILL. Les résultats expérimentaux sont comparés à des calculs de DFT réalisés sur une molécule de $\mathrm{C}_{60}$ isolée (encart gris foncé). Le premier mode intramoléculaire de symétrie $\mathrm{Hg}(1)$ du $\mathrm{C}_{60}$ est indiqué, ainsi que la zone de Gap (voir texte).

meilleures performances dans des domaines complémentaires du spectre, tout en ayant un domaine de recouvrement : ils sont donc parfaitement complémentaires et permettent des determinations de densité d'états dans une large gamme de fréquences, y compris à de très basses températures. Comme nous pouvons le voir sur la figure 5, le spectre de la GDOS comporte un grand nombre de pics, plus ou moins complexes, et s'étend jusqu'à $210 \mathrm{meV}$. Il couvre donc un domaine très vaste en énergie, conséquence de la liaison C-C extrêmement forte (l'une des plus fortes liaisons chimiques de la nature) et de la masse faible des atomes de carbone. Ces spectres sont comparés à des calculs de DFT réalisés à l'aide du logiciel $\mathrm{DMol}$ (voir cours de M. Johnson et al.) sur une molécule de $\mathrm{C}_{60}$ isolée, ce qui permet d'attribuer les différents modes. Par exemple, le premier mode de vibration intramoléculaire sera le mode $\mathrm{Hg}^{1}$, qui implique une déformation elliptique de la molécule.

On découpe généralement le spectre des vibrations internes du $\mathrm{C}_{60}$ en modes "radiaux" (30 meV < $\mathrm{E}<110 \mathrm{meV})$ et en modes "tangentiels" ( $115 \mathrm{meV}<\mathrm{E}<200 \mathrm{meV})$ où les vibrations impliquent des déformations des liaisons $\mathrm{C}-\mathrm{C}$ ("stretching"). Pour des fréquences supérieures à $30 \mathrm{meV}$, ce découpage en bande se retrouve de façon équivalente dans le spectre du graphite, alors que les spectres à basse fréquence sont très différents : ceci indique que les interactions locales sont proches dans les deux systèmes.

Il est important de constater que les spectres expérimentaux sont en très bon accord avec les calculs, ce qui justifie $a$ posteriori l'hypothèse de découplage des mouvements.

\section{Excitations inter-moléculaires du $\mathbf{C}_{60}$}

Intéressons-nous à présent à la dynamique de réseau des $\mathrm{C}_{60}$ dans la phase dite ordonnée qui apparait lorsque la température passe en dessous de $260 \mathrm{~K}$. Cette dynamique est celle des modes de vibrations "externes" - aussi appelés "inter-moléculaires" ou "de réseau", mais on se réfère dans tous les cas aux mêmes excitations. Afin de bien comprendre cette dynamique des excitations de réseau dans la phase ordonnée, il est nécessaire de fournir une description précise de la structure de cette phase. La figure 4 montre une représentation schématique de la configuration des $\mathrm{C}_{60}$ dans la phase ordonnée de groupe d'espace $\mathrm{Pa} \overline{3}$. 

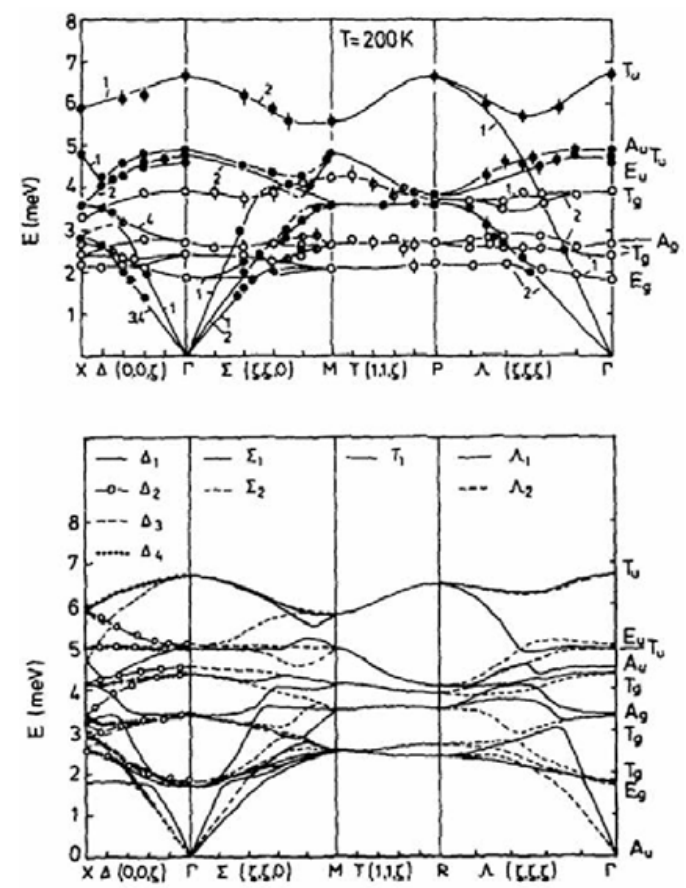

Figure 6. Haut : Courbes de dispersion mesurées à $200 \mathrm{~K}$ sur un monocristal de $\mathrm{C}_{60}$. - Bas : Résultats de calculs de courbes de dispersion avec un modèle de constantes de forces prenant en compte des interactions de van der Waals entre fullerènes. D'après la référence [13]).

Dans cette phase, les $\mathrm{C}_{60}$ cristallisent dans un système cubique simple. Bien que les molécules soient positionnées sur les sites correspondant à une maille qui serait cubique faces centrées dans le cas de molécules indiscernables, ce qui est le cas dans la phase désordonnée, la maille devient cubique simple dans le cas où elles sont discernables. Dans la phase ordonnée, les $\mathrm{C}_{60}$ peuvent être orientés de telle sorte que l'on aboutit à deux types de configurations relatives : i) la configuration nommée "P" (comme pentagone) où une double liaison d'un $\mathrm{C}_{60}$ fait face à un pentagone de la molécule voisine, et ii) la configuration nommée " $\mathrm{H}$ " (comme hexagone) où une double liaison d'un $\mathrm{C}_{60}$ fait face à un hexagone de la molécule voisine. Il en résulte qu'une maille cristalline contient 4 molécules de $\mathrm{C}_{60}$ nonéquivalents. Il y a donc 24 degrés de liberté par maille : 3 rotations et 3 translations par molécules. La dynamique du réseau des fullerènes comporte donc 24 branches de dispersion dont 3 modes acoustiques, qui peuvent être mesurées sur un mono-cristal en utilisant un spectromètre 3-axes.

Les premières mesures de courbe de dispersion des modes externes furent réalisées par Pintchovius et al. en 1992 sur le spectromètre 3-axes 2T du Laboratoire Léon Brillouin [14] sur un mono-cristal de très petite taille $\left(3 \mathrm{~mm}^{3}\right)$. Une optique focalisante fut utilisée de façon à rendre possibles les mesures sur cet échantillon dans un temps raisonnable. Les résultats expérimentaux sont montrés sur la figure 6, et sont comparés à des calculs de dynamique des réseaux qui utilisent un modèle d'interaction de type van der Waals [13].

À cause de la dégénérescence de certains de ces modes et de la résolution imparfaite de l'expérience de DIN, il n'est pas possible d'observer les 24 branches de dispersion sur le haut de la figure 6. Certaines de ces branches gardent leur caractère de translation (on les appellera "phonons"), et d'autres leur caractère de rotation (on les appellera "librations"). D' autre modes ont un caractère mixte. Il est possible de déterminer expérimentalement le caractère de chacune de ces branches grâce à la dépendance en fonction du vecteur de diffusion de l'intensité de la fonction de diffusion associée à ce mode (voir [15] 
pour de plus amples détails). Il est aussi possible d'observer des phénomènes d'anticroisement entre les branches, ce qui traduit un couplage entre certains des modes inter-moléculaires. Le mélange de caractère des modes indique un couplage entre les degrés de liberté de rotation et de translation, et ce couplage est en particulier responsable de la contraction du réseau à la mise en ordre [16]. Ces phénomènes, ainsi que l'allure générale des branches de dispersion, sont bien reproduits par le modèle proposé par Pintschovius et al. [13].

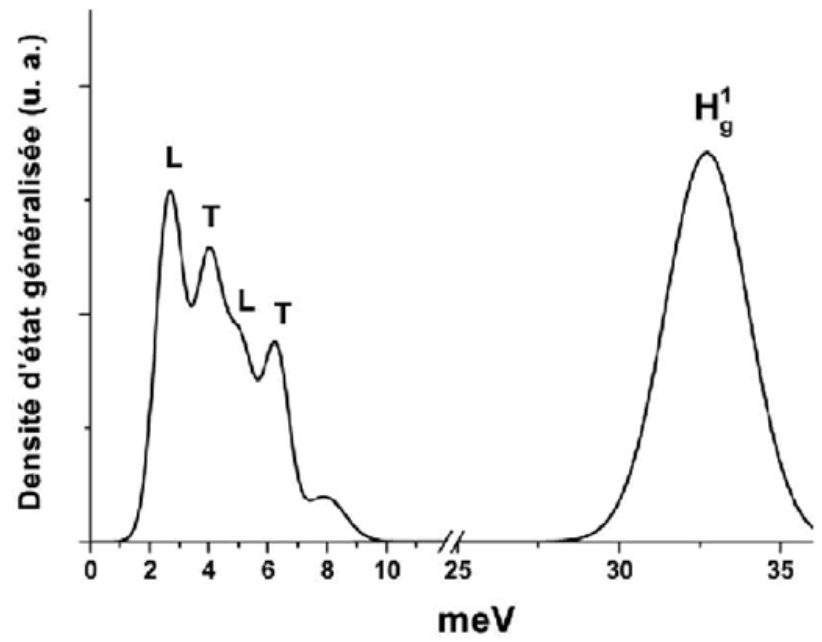

Figure 7. GDOS d'une poudre de $\mathrm{C}_{60}$ mesurée à température ambiante sur IN4C à l'ILL.

La densité d'état des modes externes peut être mesurée directement sur une poudre. La figure 7 montre le spectre mesuré sur le spectromètre IN4C dans un domaine d'énergie autour des modes externes. Le premier pic de la DOS à $2.7 \mathrm{meV}$ est attribué à la composante principale des contributions des modes associés aux librations. Il est à noter que la dépendance en fonction du vecteur de diffusion $\mathrm{Q}$ de ce mode est caractéristique du mouvement de rotation du $\mathrm{C}_{60}$. Le facteur de structure dynamique mesuré sur le spectromètre IN4C est représenté sur la figure 8. On voit apparaître des lobes intenses situés à l'énergie $\mathrm{E} \simeq 2.5 \mathrm{meV}$ et aux vecteurs de diffusion $\mathrm{Q}=3.3$ et $5.0 \AA^{-1}$. Ils correspondent aux maxima du facteur de forme associé aux mouvements de rotation de la molécule de $\mathrm{C}_{60}[4,8,15,17]$. Par la suite, on appellera souvent ce pic situé à environ $2.5 \mathrm{meV}$, "la" libration du $\mathrm{C}_{60}$.

\subsubsection{Effet de la transition ordre/désordre sur la dynamique de basse fréquence du $\mathrm{C}_{60}$}

La transition de phase ordre-désordre est du premier ordre dans le $\mathrm{C}_{60}$. À une température proche de $260 \mathrm{~K}$, les molécules de $\mathrm{C}_{60}$ se mettent en rotation quasi-libre ${ }^{5}$. L'effet le plus important dans la dynamique des fullerènes porte bien entendu sur la libration. Celle-ci perd son caractère oscillatoire (fluctuation périodique des orientations dans le temps) au profit d'un caractère diffusif. Sur la figure 9 les lobes dans le spectre $S(Q, \omega)$ sont perdus au profit d'un excès d'intensité dans les pieds de la raie élastique - appelée intensité "quasi-élastique" (QE) - et qui est associée au caractère diffusif des fonctions de corrélations d'orientations des molécules.

\footnotetext{
5 On utilise ici le terme "quasi" dans le sens où il existe des correlations d'orientations entre molécules voisines. Cependant, leur temps caractéristique et leur portée sont tellement faibles qu'elles peuvent être négligées en première approximation. Par la suite nous parlerons de rotations libres et négligerons les correlations d'orientations dans la phase désordonnée.
} 


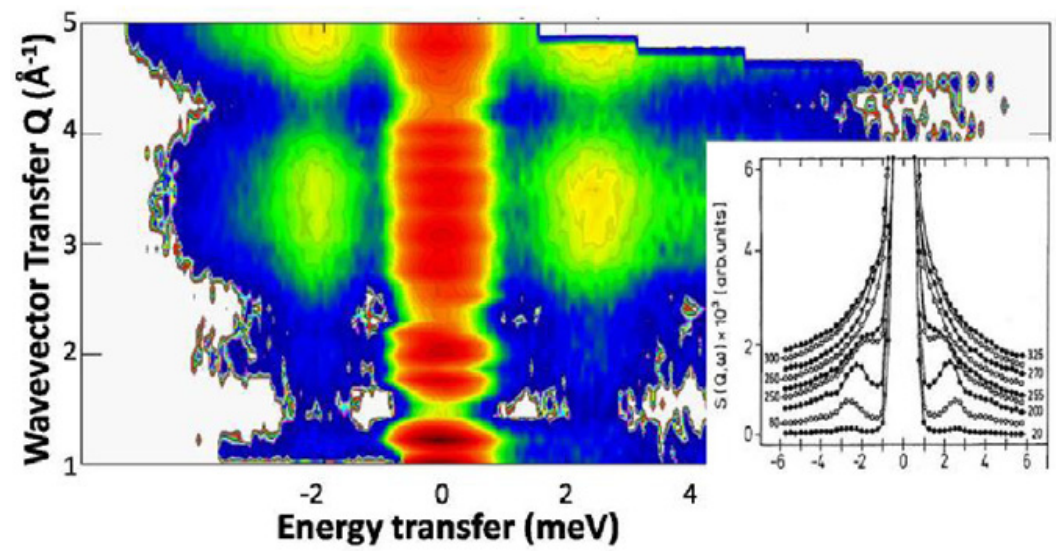

Figure 8. Facteur de structure du $\mathrm{C}_{60}$ dans le domaine basse fréquence mesuré sur IN4C, dans la phase ordonnée à $\mathrm{T}=220 \mathrm{~K}$. - Bas droit : Evolution du signal des rotations de basse fréquence en fonction de la température (d'après [17]).

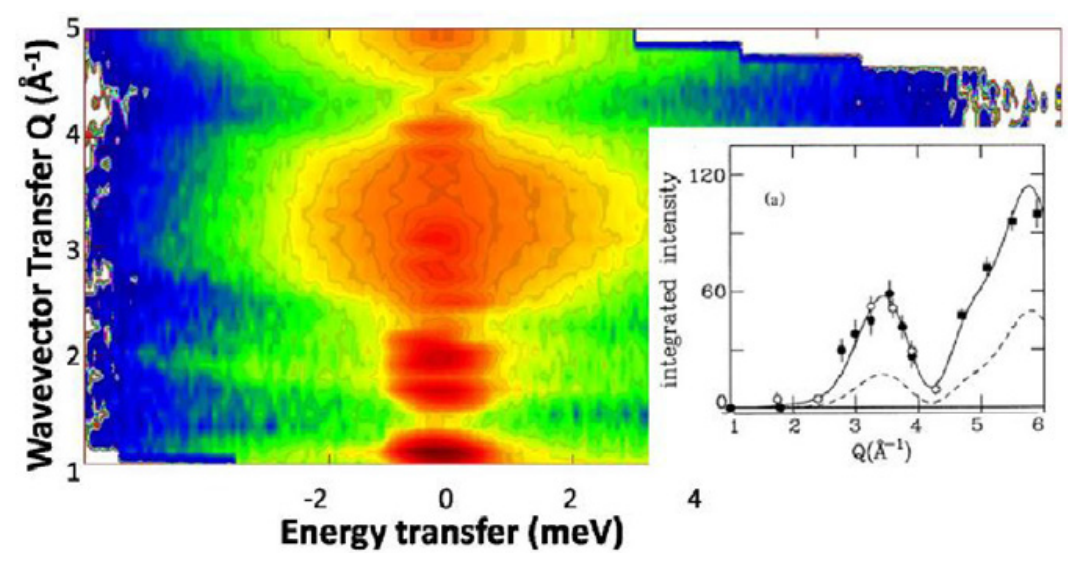

Figure 9. Facteur de structure du $\mathrm{C}_{60}$ dans le domaine basse fréquence mesuré sur IN4C, dans la phase désordonnée à $\mathrm{T}=300 \mathrm{~K}-$ Bas droit : Evolution de l'intensité intégrée du signal quasiélastique en fonction du vecteur de diffusion Q. La ligne continue est issue d'un modèle de rotation isotrope du $\mathrm{C}_{60}$. (d'après [8]).

On peut aussi observer sur la partie basse droite de la figure 8 que le signal QE, nettement visible pour les températures supérieures à $270 \mathrm{~K}$, se structure dès $255 \mathrm{~K}$ pour donner naissance au pic de la libration. Ce dernier est séparé du pic élastique, et sa position semble s'écarter de l'élastique au fur et à mesure que la température baisse. L'amollissement apparent de ce mode au voisinage de la transition a d'abord été considéré comme une preuve de l'anharmonicité du potentiel de libration des fullerènes, avant d'avoir finalement été attribué à un effet plus subtil : il est en effet lié au changement de la proportion des $\mathrm{C}_{60}$ en orientation $\mathrm{H}$ et $\mathrm{P}$ dans la phase ordonnée en fonction de la température [18], la fréquence de la libration de l'orientation P (la plus stable) étant plus élevée que celle de la configuration $\mathrm{H}$.

La partie gauche de la figure 10 montre l'évolution du taux d'occupation des sites du cristal par des $\mathrm{C}_{60}$ en orientation $\mathrm{P}$ en fonction de la température. Ces résultats ont été obtenus par diffraction des neutrons à haute résolution. On observe qu'entre $255 \mathrm{~K}$ et $90 \mathrm{~K}$, cette proportion augmente de manière continue, puis reste constante en dessous de $90 \mathrm{~K}$. Cette discontinuité se retrouve dans la courbe traçant 

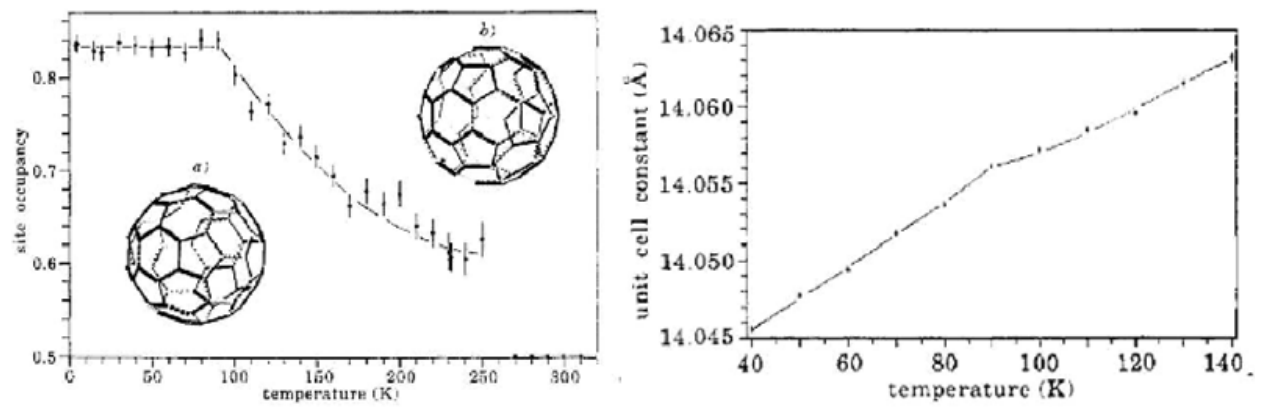

Figure 10. Gauche : Évolution de la proportion de $\mathrm{C}_{60}$ en orientation $\mathrm{P}$ (notée (a)) par rapport à l'orientation $\mathrm{H}$ avec la température - Droite : Évolution du paramètre de maille du cristal de $\mathrm{C}_{60}$ avec la température. D'après la référence [19].

le paramètre de maille du cristal en fonction de la température où l'on observe un changement de pente dans la dilatation du cristal à $90 \mathrm{~K}$ (figure 10 à droite). La raison de cette discontinuité - qui se retrouve aussi dans l'évolution de la conductivité thermique du matériau - est une nouvelle transition dynamique. En dessous de $90 \mathrm{~K}$, les mouvements de réorientations entre phase $\mathrm{P}$ et $\mathrm{H}$ cessent, et le système passe donc dans un état appelé verre orientationnel où la proportion de $\mathrm{C}_{60}$ en orientation $\mathrm{P}$ reste fixée à $83.5 \%$.

Dans la phase désordonnée, la perte des modes de libration ainsi que l'augmentation de la symétrie du cristal (on est maintenant dans un réseau cubique à faces centrées $\mathrm{Fm} \overline{3} \mathrm{~m}$ ) simplifie grandement le spectre des modes externes. En effet, il ne reste que 3 degrés de liberté par molécule - ceux de rotation ont disparus - et une molécule par maille. Les courbes de dispersion se résument à 3 branches acoustiques, comme représenté sur la figure 11. Elles peuvent être modélisées de manière très correcte par un modèle simple, où chaque $\mathrm{C}_{60}$ est assimilé à un atome de gaz noble en interaction avec les autres au moyen d'un tenseur de constantes de forces à 3 paramètres ajustables [13,15].

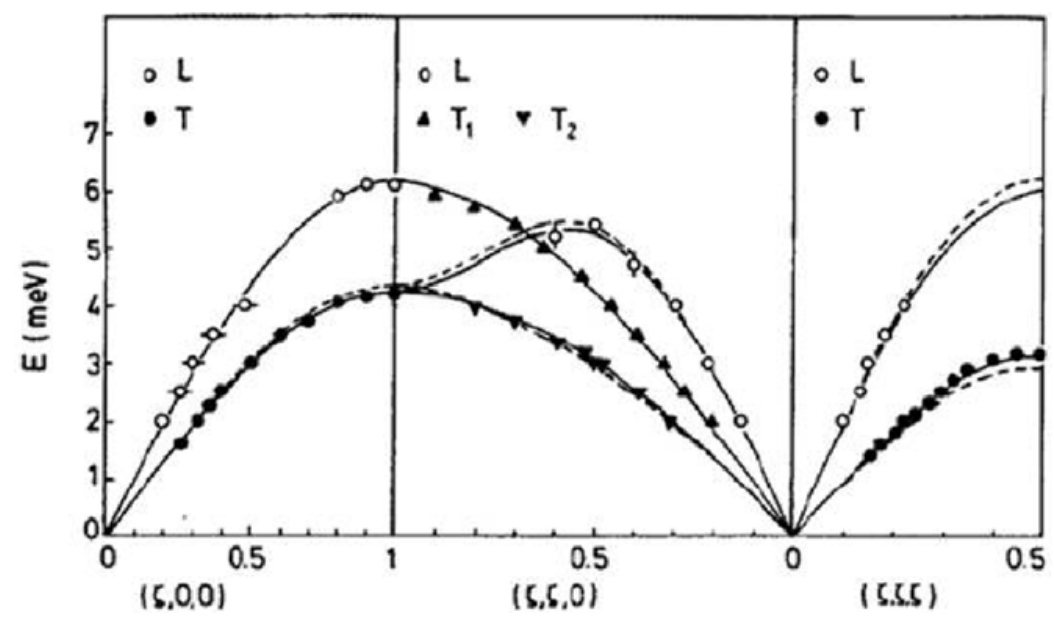

Figure 11. Courbes de dispersion des modes externes d'un monocristal de $\mathrm{C}_{60}$ dans la phase désordonnée. Les lettres $\mathrm{L}$ et $\mathrm{T}$ indiquent les branches longitudinales et transverses respectivement. La ligne continue est issue d'un calcul basé sur un modèle de constante de forces à 3 paramètres. Les lignes en tirets sont les courbes de dispersion des phonons du Krypton (d'après [17]). 


\subsection{Exemple de cristal moléculaire à 2 motifs : le fullerène-cubane $\mathrm{C}_{60} \cdot \mathrm{C}_{8} \mathrm{H}_{8}$}

Depuis 2005, un nouveau matériau a été synthétisé sur la base de fullerènes intercalés : il s'agit du fullerène-cubane, dans lequel des molécules de cubane $\left(\mathrm{C}_{8} \mathrm{H}_{8}\right)$ s'intercalent dans les sites octaédriques du réseau $\mathrm{CFC}$ des $\mathrm{C}_{60}$ (voir figure 12). Le paramètre de maille de la structure résultante est dilaté par rapport à celui du $\mathrm{C}_{60}$ pur à $300 \mathrm{~K}(14.74 \AA$ contre $14.15 \AA)$.
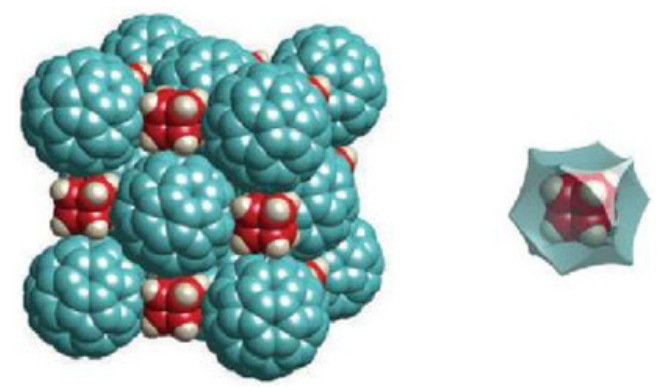

Figure 12. Représentation schématique d'un cristal de fullerène-cubane (à gauche) et détail de la forme du site octaédrique inter-fullerènes autour d'une molécule de cubane. D'après la référence [20].

Il est remarquable de constater que l'augmentation du paramètre de maille causée par l'intercalation du cubane est plus faible que la dimension latérale de la molécule de cubane. Cela implique que la forme des molécules de cubane présente une complémentarité avec le cristal de $\mathrm{C}_{60}$ et peut s'y mélanger intimement.

La figure 13 montre la densité d'état généralisée mesurée sur le spectromètre IN4C à $300 \mathrm{~K}$. Le spectre peut être découpé en 3 domaines (LF, MF et HF), chacun étant associé à un certain type de dynamique. Les molécules de cubane comportent chacune 8 atomes d'hydrogène. La section efficace de diffusion sera donc dominée par la contribution incohérente forte due aux molécules de cubane, et ainsi, les pics les plus intenses du spectre seront associés à cette molécule.

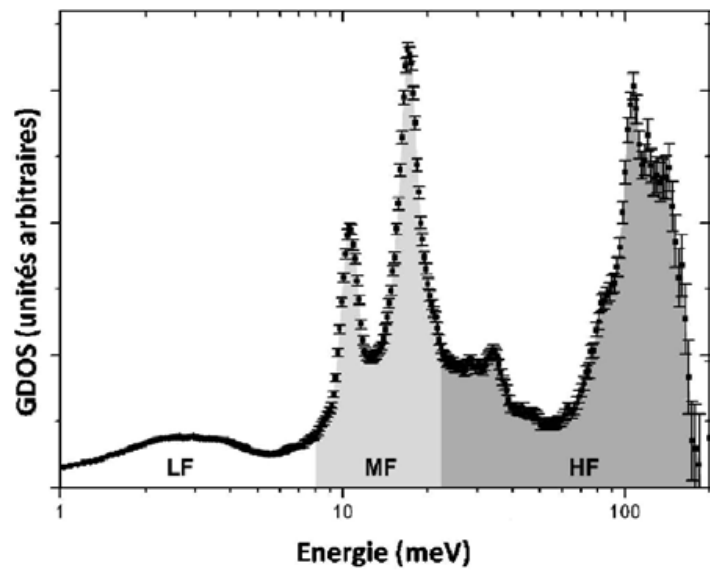

Figure 13. Densité d'état généralisée d'une poudre de fullerène-cubane, mesurée sur le spectromètre IN4C dans la phase désordonnée. Les domaines LF, MF, et HF repèrent les zone basses fréquences, fréquences intermédiaires et hautes fréquences respectivement. 
Les modes moléculaires du cubane ont été étudiés par Yildirim et al. [21] : ils se trouvent dans la bande HF (à l'exception des modes de déformation de la liaison C-H qui se trouvent à plus haute fréquence) et forment une structure très intense entre $70 \mathrm{meV}$ et $170 \mathrm{meV}$.

Des expériences de $\mathrm{RMN}$ ont permis de montrer que les molécules $\mathrm{C}_{60}$ présentent un désordre orientationnel à température ambiante - équivalent à la phase désordonnée du $\mathrm{C}_{60}$ cristallin pur - alors que les molécules de cubane restent figées autour d'orientations bien déterminées. Cette opposition dans la dynamique des molécules de ce système lui a valu le qualificatif de "rotor-stator" dans sa phase désordonnée.

Une transition structurale vers une phase orthorhombique, moins symétrique, a été mise en évidence dans ce matériau par diffraction des rayons X à une température de 140 K [20]. La diffusion inélastique des neutrons a permis de montrer que les modes de la bande MF et de la bande LF sont particulièrement affectés par la transition. La rotation libre $\mathrm{du} \mathrm{C}_{60}$ se transforme en une libration au dessous de $140 \mathrm{~K}$, dont la fréquence $\left(\sim 2.2 \mathrm{meV}\right.$ ) est inférieure à celle du $\mathrm{C}_{60}$ pur (voir la figure 14, Gauche). Cet amollissement s'observe aussi dans des mesures de capacité calorifique [23]. Il est à noter que, contrairement au cas du $\mathrm{C}_{60}$ pur, la fréquence de la libration des fullerènes ne varie pas avec la température. Ceci suggère qu'il n'existe qu'un seul type d'arrangement dans la phase ordonnée, ou que ces arrangements possèdent des fréquences de libration très proches.

La partie droite de la figure 14 montre que la bande MF comporte deux contributions dont l'intensité suggère qu'elles sont associées aux molécules de cubane : un pic à $11 \mathrm{meV}$ et un à $17.8 \mathrm{meV}$. En
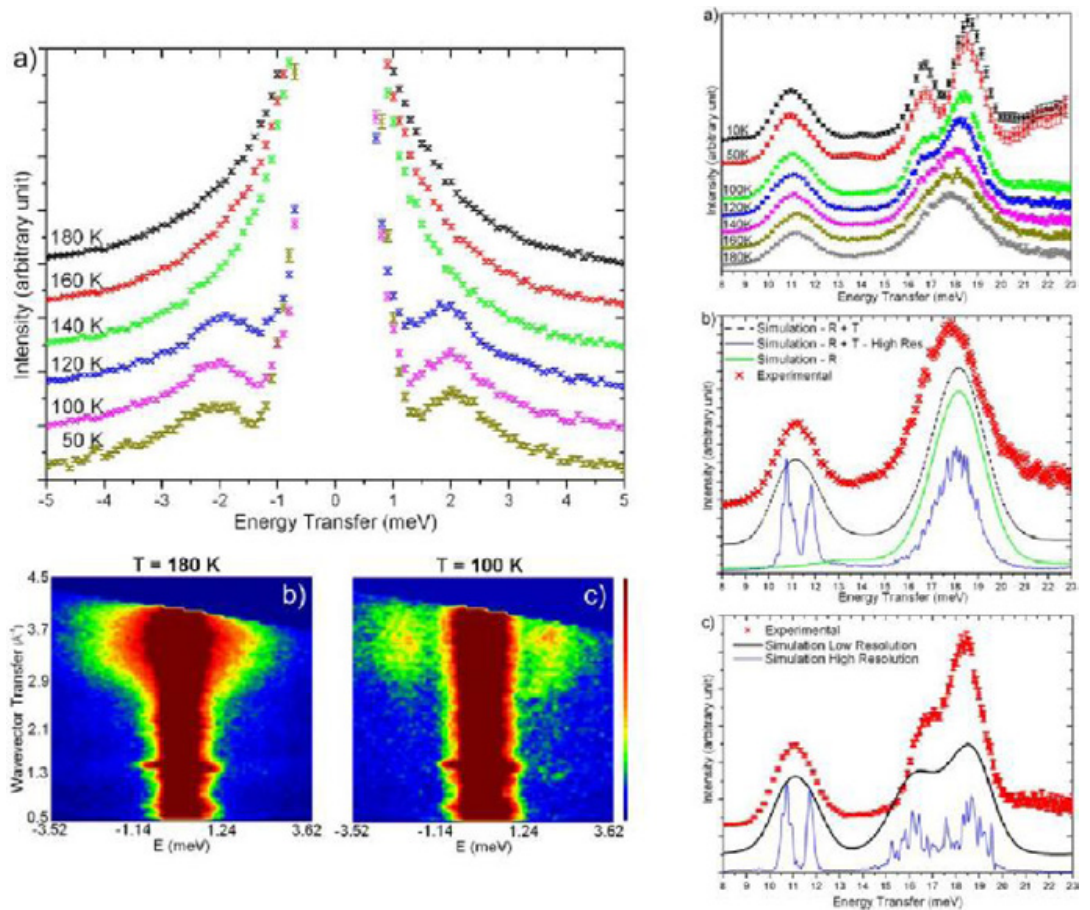

Figure 14. Gauche : évolution de $\mathrm{S}(\mathrm{Q}, \omega)$ en fonction de la température. a) spectres en énergie pour $\mathrm{Q} \sim 3.3 \AA^{-1}$ pour différentes températures au dessus et au dessous de la transition ordre-désordre $\left(\mathrm{T}_{c}=140 \mathrm{~K}\right)$; b) et c) carte $\mathrm{S}(\mathrm{Q}, \omega)$ pour $\mathrm{T}=180 \mathrm{~K}\left(\mathrm{~T}>\mathrm{T}_{c}\right)$ et $\mathrm{T}=100 \mathrm{~K}\left(\mathrm{~T}<\mathrm{T}_{c}\right)$ respectivement. - Droite $:$ a) Évolution de la bande $\mathrm{MF}$ en fonction de la température; b) et c) comparaison du spectre expérimental avec la GDOS calculée par dynamique moléculaire dans la phase désordonnée et ordonnée respectivement. Les spectres simulés notés R+T sont calculés à partir de molécules rigides prenant en compte les mouvements de rotation et de translation de la molécule. Les spectres notés R ne prennent en compte dans les calculs que les mouvements de rotation (reproduit de [22]). 
abaissant la température sous $\mathrm{T}_{c}$, le pic à haute fréquence se sépare en deux contributions $(16.5 \mathrm{meV}$ et $18.5 \mathrm{meV}$ ) dont le rapport d'intensité est $1 / 2$.

L'attribution des pics s'est faite par le biais de calculs de dynamique moléculaire, qui nous ont permis de calculer les trajectoires des atomes en fonction du temps. De ces trajectoires, nous pouvons déduire les fonctions neutroniques, dont la densité d'état généralisée ${ }^{6}$. Il est aussi possible de rigidifier les molécules dans la simulation - ce qui permet de rendre les calculs plus rapides - et les résultats obtenus ne concernent alors que les degrés de liberté externes de la molécule (les spectres correspondants sont notés "R+T" dans la partie droite de la figure 14). En outre, il est aussi possible d'éliminer les mouvements des centres de masses des molécules lors du calcul des fonctions neutroniques. Ainsi, on élimine du spectre les contributions des translations des molécules, et celles liées aux rotations peuvent être isolées (les spectres correspondants sont notés "R" dans la partie droite de la figure 14). Ceci nous a permis d'associer le massif à $17.8 \mathrm{meV}$ aux 3 librations du cubane, alors que le pic à $11 \mathrm{meV}$ concerne lui les vibrations de son centre de masse.

Dans la phase désordonnée $\left(\mathrm{T}>\mathrm{T}_{c}\right.$ ), l'environnement du cubane est isotrope. Toutes les molécules de $\mathrm{C}_{60}$ qui l'entourent étant équivalentes, il est alors normal que toutes les librations soient dégénérées (un seul pic de libration à $17.8 \mathrm{meV}$ ). Lorsque l'on abaisse la température sous la température critique $\mathrm{T}_{c}$, il apparaît dans le massif des librations un doublet résultant de la brisure de symétrie. Le rapport 1/2 des intensités des deux pics du doublet suggère que deux directions de l'espace sont équivalentes.

Le pic à $11 \mathrm{meV}$ quant à lui n'est pas sensible à la mise en ordre du système. Ce pic domine le domaine des phonons de la GDOS où les contributions des modes - optiques et acoustiques - du réseau sont attendues.

Le cristal de fullerène-cubane possède une structure CFC à température ambiante, et le motif de la maille élémentaire contient donc une molécule de $\mathrm{C}_{60}$ (de masse $M_{C_{60}}=720 \mathrm{uma}$ ) et une molécule de cubane (de masse $M_{C_{8} H_{8}}=104$ uma). Les courbes de dispersion de phonon possèdent donc 6 branches -3 acoustiques et 3 optiques.

Il est intéressant et éducatif de comprendre pourquoi le pic unique à $11 \mathrm{meV}$ domine le spectre dans le domaine des phonons. On renvoie pour cela le lecteur au chapitre I (section 3.3.2) ainsi qu'à l'équation 9.115 du chapitre III. Afin d'appliquer ce formalisme au cas du fullerène cubane, supposons le cristal composé de deux molécules $\bigcirc$ et $\square$, de masses respectives $M_{\bigcirc}=M_{C_{60}}$ et $M_{\square}=M_{C_{8} H_{8}}$. Supposons de plus que leurs sections efficaces de diffusion incohérente sont $\sigma_{i n c}(\bigcirc)$ et $\sigma_{i n c}(\square)$, et étudions les phonons de ce cristal moléculaire $\{\bigcirc, \square$ \} en supposant ces molécules rigides (on omet ses degrés de liberté interne). La formule 9.115 du chapitre III s'écrit alors :

$$
\left(\frac{\partial^{2} \sigma}{\partial \Omega \partial E_{f}}\right)_{i n c}^{1-p h(+)}=\frac{k_{f}}{k_{i}} \sum_{\kappa \in\{\bigcirc, \square\}} \frac{\sigma_{i n c}(\kappa)}{8 \pi M_{\kappa}} e^{-2 W_{k}(\vec{Q})} \sum_{j, \vec{k}} \frac{\left|\vec{Q} \cdot \vec{e}_{j}(\kappa \mid \vec{k})\right|^{2}}{\omega_{j}(\vec{k})}\left[1+n\left(\omega_{j}(\vec{k})\right)\right] \delta\left(\omega-\omega_{j}(\vec{k})\right)
$$

Dans notre cas, nous avons $\sigma_{\text {inc }}(\bigcirc)=0$., et cette équation peut se re-écrire :

$$
\left(\frac{\partial^{2} \sigma}{\partial \Omega \partial E_{f}}\right)_{i n c}^{1-p h(+)}=\frac{k_{f}}{k_{i}} \frac{\sigma_{i n c}(\square)}{8 \pi M_{\square}} e^{-2 W_{\square}(\vec{Q})} \sum_{j, \vec{k}} \frac{\left|\vec{Q} \cdot \vec{e}_{j}(\square \mid \vec{k})\right|^{2}}{\omega_{j}(\vec{k})}\left[1+n\left(\omega_{j}(\vec{k})\right)\right] \delta\left(\omega-\omega_{j}(\vec{k})\right)
$$

Comme discuté au chapitre I (section 3.3.2) dans le cas de la chaîne linéaire diatomique, la différence de masse importante entre les deux molécules est responsable de la très faible dispersion des modes optiques, et donc de la contribution très importante de ces modes à la densité d'état. Cette différence de masse est aussi responsable de la quasi-immobilité des molécules de masse faible (ici le cubane) pour les modes acoustiques de bord de zone, soit $\vec{e}_{a} c\left(\square \mid \vec{k}_{\in B Z}\right)=0$. D'après l'équation 2.2, ceci implique que l'intensité des modes acoustiques de bord de zone sera très faible dans le spectre de la GDOS, et qu'ainsi le spectre des phonons du fullerène-cubane sera dominé par une seule contribution intense provenant

\footnotetext{
${ }^{6}$ Par la transformée de Fourier des fonctions d'autocorrélation des vitesses pondérées par les longueurs de diffusion des atomes.
} 

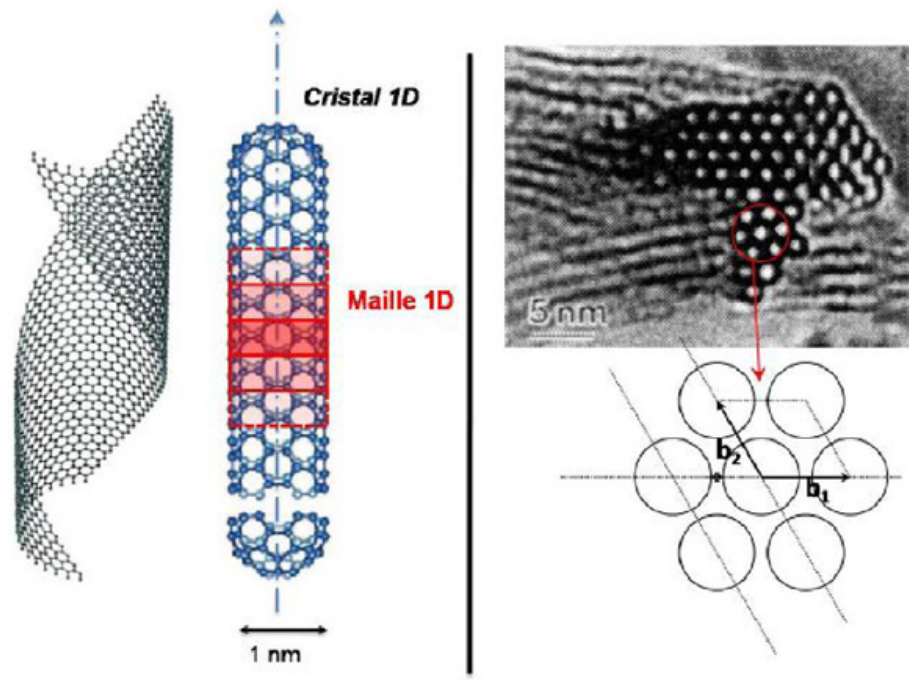

Figure 15. Gauche : Schéma de la structure d'un nanotube monofeuillet isolé et définition de sa maille élémentaire - Droite : Organisation en faisceau de tubes parallèles et définition du réseau moléculaire 2D (l'image de microscopie électronique à transmission est issue de [25]).

des modes optiques. La non-dispersité des modes optiques fait penser aux contributions des modes de "hochet" (vibration dans des cages) qui sont présentées dans la contribution de H. Mutka à cet ouvrage.

En conclusion, il apparaît clairement que la transition structurale observée à $\mathrm{T}_{c}=140 \mathrm{~K}$ s'accompagne d'une transition dynamique où la phase rotor-stator est perdue au profit d'une phase où les mouvements de rotation des deux type de molécules sont à caractère oscillatoire. Cette température de transition est très faible si on la compare à celle qui existe dans la phase cristalline du $\mathrm{C}_{60}$ pur $(260 \mathrm{~K})$. Le système des $\mathrm{C}_{60}$ se trouve fortement lubrifié par la présence des molécules de cubane, dû à la complémentarité des formes (concave et convexe) des molécules de $\mathrm{C}_{60}$ et de cubane, qui s'imbriquent l'une dans l'autre. Les molécules de cubane jouent ainsi le rôle de "roulement moléculaire" dans le système. La transition de phase ne semble mettre en jeu principalement que les degrés de liberté de rotations des $\mathrm{C}_{60}$ et du cubane.

\section{LES VIBRATIONS DANS UN CRISTAL MOLÉCULAIRE 2D ET ATOMIQUE $1 D$ : LES FAISCEAUX DE NANOTUBES DE CARBONE}

\subsection{Un nanotube issu de l'enroulement d'une feuille de graphène}

Il existe deux grandes familles de nanotubes de carbone : les nanotubes de carbone monofeuillets $(\mathrm{SWNT})$ et les nanotubes de carbone multifeuillets $(\mathrm{MWNT})^{7}$. Les SWNT peuvent être considérés comme le résultat de l'enroulement d'un feuillet de graphène sur lui-même, de manière à former un cylindre d'une longueur d'environ $1 \mu \mathrm{m}$ pour un diamètre de l'ordre du nanomètre. Suivant la manière dont la feuille est enroulée, on aboutit à des structures atomiques différentes (on parlera aussi d'hélicités ou de chiralités différentes) qui présentent des propriétés électroniques distinctes. On peut ainsi obtenir des nanotubes métalliques ou semi-conducteurs (pour plus de détails, consulter par exemple le chapitre 4 de [24]).

\footnotetext{
${ }^{7}$ Nous gardons dans ce cours le sigle anglais SWNT pour "Single Wall NanoTube" car il est abondamment utilisé dans la littérature.
} 
Quelle que soit la manière dont est enroulé le feuillet de graphène, il est toujours possible de définir une maille le long du grand axe du tube ${ }^{8}$, et les SWNT peuvent donc en ce sens être considérés comme des cristaux uni-dimensionnels (voir figure 15) que nous qualifierons d' "atomiques" étant donné que 2 mailles adjacentes sont reliées par des interactions covalentes.

Les SWNT se regroupent spontanément en faisceaux sous l'effet des forces de van der Waals intertubes. Ces faisceaux peuvent contenir plusieurs dizaines de tubes et forment dans le plan de section un réseau hexagonale (voir figure 15, partie droite). Aucune corrélation longitudinale entre deux tubes différents mais du même faisceau n'a jamais été mise en évidence. C'est pourquoi nous qualifierons ce système d"' atomique 1D" et de "moléculaire 2D" suivant la direction étudiée.

\subsection{Excitations dans les nanotubes de carbone}

La maille unidimensionnelle d'un nanotube de carbone contient - au minimum - un nombre $\mathrm{N}$ de quelques dizaines d'atomes de carbone. Le nombre de branches $3 \mathrm{~N}$ de courbes de dispersion dans la direction longitudinale est donc relativement grand, ce qui rendrait leur mesure très complexe même s'il était possible de synthétiser des mono-cristaux.

Pour des tubes isolés, 4 branches possèdent une fréquence nulle en centre de zone de Brillouin. Il s'agit des trois modes acoustiques (1L, 2T) ainsi que de la rotation autour de l'axe du tube. Dès lors que les tubes s'auto-organisent en faisceau, la rotation devient une libration de basse fréquence qui est l'un des premiers modes optiques du système. Les courbes de dispersion - et la densité d'état qui leur est associée - calculées pour un nanotube de diamètre 14 A, isolé et en faisceau, sont présentées sur la figure 16.
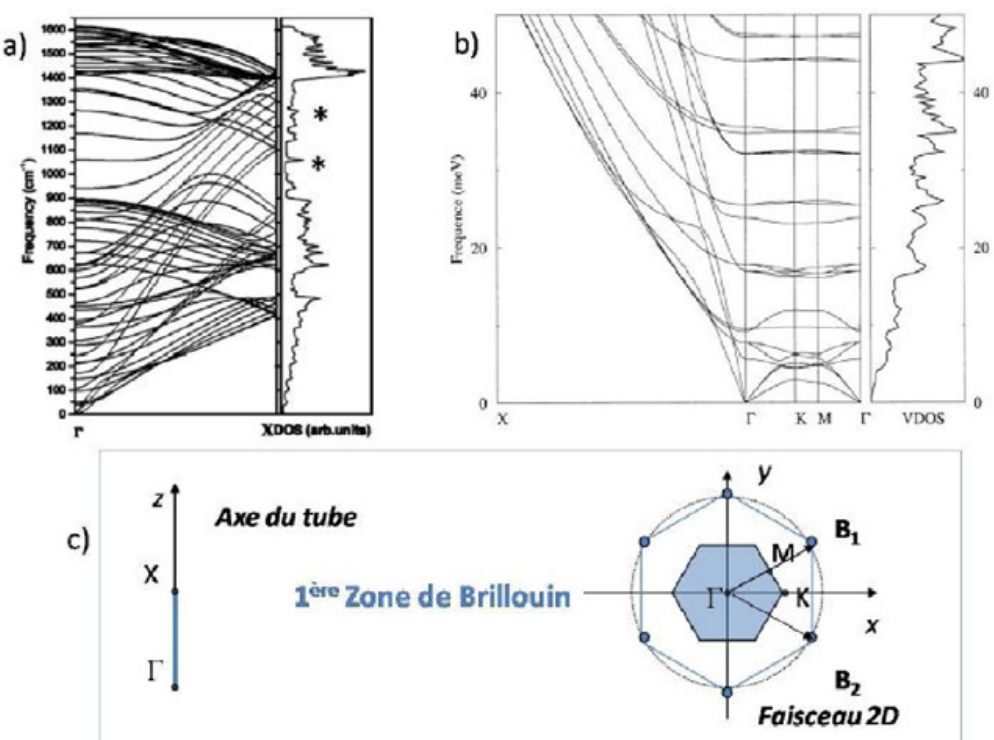

Figure 16. a) : Courbes de dispersion de phonons d'un nanotube isolé de diamètre $14 \AA$ (d'après la référence [26]) - b) Partie basse fréquence des courbes de dispersion de phonons d'un nanotube de diamètre 14 Å organisé en faisceau (d'après la référence [24]) - c) La première zone de Brillouin d'un nanotube isolé se limite à un segment de droite parallèle à la direction longitudinale (gauche), tandis qu'elle prend la forme d'un cylindre de section hexagonale en faisceau (droite). Les étoiles sur la figure a) indiquent des contributions spécifiques aux nanotubes qui semblent avoir été mesurées expérimentalement (voir figure 17 courbe c)).

${ }^{8}$ Qu'on appellera axe longitudinal. 
La complexité des courbes de dispersion du tube isolé se reflète dans la densité d'état par un profil très haché. Ceci est dû à la multitude de singularités de van Hove 1D, issues du repliement des branches de dispersion du graphène, en centre et en bord de zone de Brillouin du tube ${ }^{9}$. À basse fréquence, le spectre est dominé par les modes radiaux - ces modes impliquent la déformation de la forme cylindrique du tube. La fréquence de ces premiers modes optiques peut être très basse, au point de s'hybrider avec les modes de faisceau. Ainsi, contrairement au cas du $\mathrm{C}_{60}$, on ne peut pas faire de séparation nette entre modes moléculaires et modes de réseaux dans les nanotubes. Le plus célèbre de ces modes de déformations radiales est le mode de respiration, de symétrie $\mathrm{Hg}$ et actif en Raman, dont la fréquence est reliée au diamètre de façon linéaire [27]. Pour des tubes de diamètre $14 \AA$, ce mode est mesuré à $180 \mathrm{~cm}^{-1}$ (une représentation de ce mode se trouve sur la figure 17).

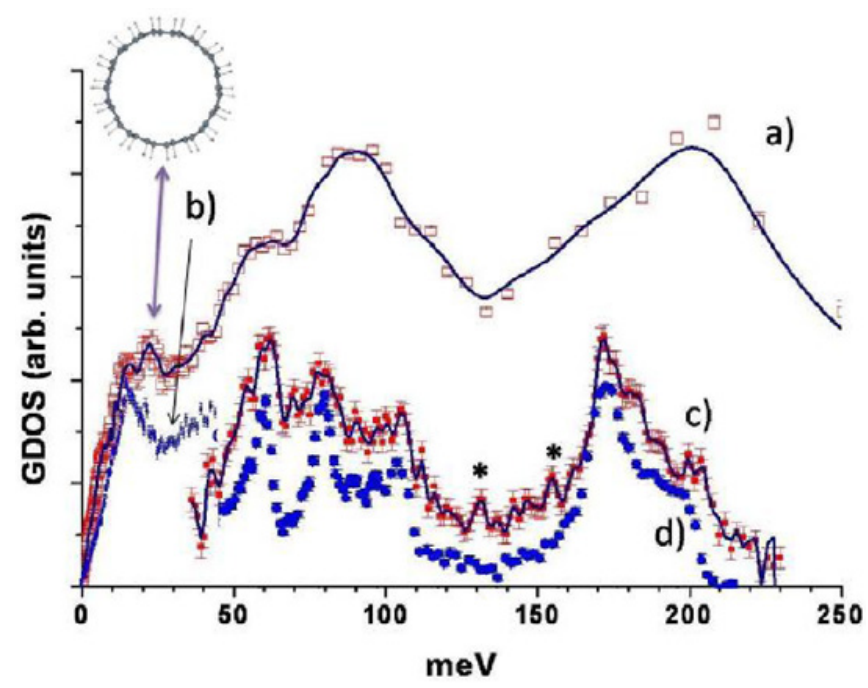

Figure 17. a) Densité d'état généralisée (GDOS) d'une poudre de nanotubes de carbone monofeuillets de diamètre 14 Å, mesurée sur le spectromètre IN6 à $300 \mathrm{~K}$ et c) mesurée sur le spectromètre IN1BeF à $10 \mathrm{~K}$ - b) et d) Idem pour le graphite. Les étoiles indiquent des singularités de van Hove qui peuvent être les équivalentes de celles calculées dans la figure 16. Le mode de respiration radial est représenté. Un pic à $\sim 20 \mathrm{meV}$ dans la GDOS lui est attribué en accord avec les résultats issus de la spectroscopie Raman effectuée sur le même échantillon.

L'étude de la dynamique des nanotubes de carbone par diffusion neutronique est plus délicate que celle des fullerènes $\mathrm{C}_{60}$, car les échantillons présentent une cristallinité bien moindre ainsi qu'une distribution de paramètres structuraux (diamètre, taille des faisceaux, pureté...) qui rendent l'interprétation des données difficile. La technique la plus utilisée pour l'étude de ces systèmes est la spectroscopie Raman qui tire profit du caractère résonnant de sa section efficace pour photo-sélectionner un diamètre de tube dans l'échantillon (en fonction de la longueur d'onde du laser incident, voir le chapitre écrit par J.-L. Sauvajol et al. de la référence [24] pour plus de détail). Le phénomène de résonance est tellement puissant qu'il permet d'étudier un tube unique.

Du point de vue de la structure atomique, un nanotube de carbone est très proche d'un feuillet de graphène, et on peut donc s'attendre à ce que la densité d'état d'un échantillon de nanotubes de carbone présente de fortes similitudes avec celle d'un échantillon de graphite. Ceci se vérifie expérimentalement : la figure 17 présente une comparaison entre les GDOS de ces deux types d'échantillons (voir aussi [28]).

${ }^{9}$ Dans un système unidimensionnel, les contributions de bord de zone et de centre de zone sont en nombre égal. 
La densité d'états expérimentale des SWNT résulte de la combinaison des données obtenues sur deux spectromètres de l'ILL (IN6 et IN1BeF) complémentaires dans la gamme d'énergie sondée.

On peut remarquer que les profils expérimentaux des GDOS des nanotubes et du graphite sont effectivement très similaires dans le domaine haute fréquence, mais de petites différences sont néanmoins identifiables. Tout d'abord, dans les spectres c) et d) de la figure 17, de petits pics sont visibles dans la GDOS des SWNT sur toute la gamme de fréquence, alors qu'ils n'apparaissent pas dans la GDOS du graphite. Ces pics correspondent aux singularités de van Hove 1D et sont situés aux fréquences des modes de bord et/ou de centre de zone de Brillouin. Ces contributions sont particulièrement bien reproduites par les calculs de DFT réalisés par Ye et al. [26] et représentés sur la figure 16. Enfin, la GDOS des SWNT est plus importante que celle du graphite dans la gamme des énergies inférieures à $40 \mathrm{meV}$. Cet excès est dû à la présence de modes spécifiques des nanotubes de carbone, tels que les modes de respiration radiaux (RBM pour "radial breathing modes") illustrés sur la figure 17, qui dépendent du diamètre des tubes : ils n'ont évidemment pas d'équivalent dans le cas du graphite.

Les modes de faisceau sont très difficiles à distinguer dans la GDOS des nanotubes. Leur hybridation avec les premiers modes optiques des tubes, ainsi que le nombre relativement faible de tubes par faisceau, rend leur contribution large et difficile à isoler. Elle apparaît néanmoins aux plus basses fréquences du spectre $(\mathrm{E}<1.5 \mathrm{meV})$, dans le domaine de Debye de la GDOS, dominé par les modes acoustiques et sensible à la dimensionnalité du système (voir [29]). Dans ce domaine, on trouve une dépendance linéaire de la GDOS avec la fréquence, caractéristique d'un réseau bidimensionnel, et en accord avec la dépendance quadratique de la $C_{p}(T)$ en fonction de $\mathrm{T}$, pour $\mathrm{T}<100 \mathrm{~K}$, mesurée sur les mêmes échantillons [30,31]. La figure 16b) montre les courbes de dispersion d'un faisceau de nanotubes dans les directions longitudinale $(\Gamma-X)$ et transverse $(\Gamma-K-M-\Gamma)$. La pente des modes acoustiques dans la direction longitudinale étant très forte, leur contribution à la densité d'état sera négligeable. Dans cette région du spectre, le réseau pertinent est donc un réseau bidimensionel lié à l'arrangement des tubes en faisceaux.

\section{LES PETITS-POIS DE CARBONE : CHAÎNES DE C $0_{0}$ 1D CONFINÉES DANS LES NANOTUBES MONOFEUILLETS}

Le dernier système que nous évoquerons dans ce chapitre est un composé d'insertion des nanotubes, et combine les nanotubes de carbone monofeuillets et les fullerènes $\mathrm{C}_{60}$ en une structure appelée "peapods"10. Ces structures, révélées par microscopie électronique, doivent leur nom à leur structure qui évoque des petits pois à l'intérieur de leur cosse. Dans la suite de cet exposé, nous utiliserons donc le terme "peapods" pour désigner cette structure mixte entièrement constituée de carbone.

Les premières observations de peapods ont eu lieu en 1998 [32]. Comme souvent, la synthèse dont ont été issus ces premiers peapods n'était pas destinée à leur production, et ils ont été observés par hasard en microscopie électronique à transmission (MET). L'engouement suscité par cette découverte a été très important, et un an plus tard était publié un mode de synthèse [33] permettant de produire ces objets en quantités suffisantes pour des études plus poussées. Avec l'amélioration croissante du contrôle sur le diamètre des nanotubes produits, des méthodes de synthèse de peapods de très haute qualité (taux de remplissage élevés, haute cristallinité des faisceaux, faible taux d'impuretés) ont pu être mises au point [34]. La figure 18 montre une photographie prise en MET de haute résolution d'un de ces échantillons de grande pureté. On peut constater que le taux de remplissage apparaît comme très important et que les nanotubes remplis se regroupent en faisceaux contenant un grand nombre de tubes. Ces caractéristiques structurales ont été depuis confirmées par diffraction des rayons X [35,36].

10 "peapods" = petits pois en anglais. 


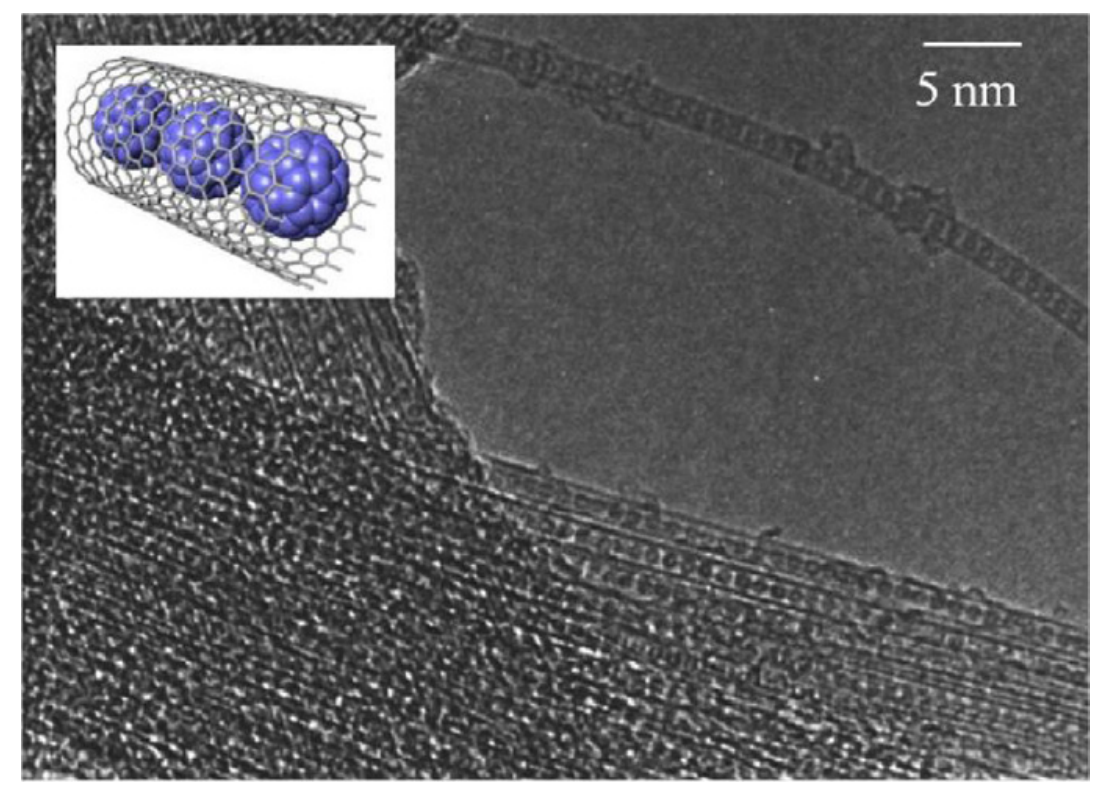

Figure 18. Image de microscopie électronique à transmission d'un échantillon de peapods. Une représentation schématique de la structure moléculaire des peapods est encadrée à gauche. D'après Kataura et al.

Il est intéressant d'étudier dans quelle mesure le confinement des fullerènes sous la forme d'une chaîne 1D modifie leurs propriétés dynamiques. Dans ce sous-paragraphe, nous allons donner un aperçu des principaux résultats obtenus en diffusion neutronique sur la dynamique de ces matériaux.

Dans les peapods, la masse des $\mathrm{C}_{60}$ ne constitue qu'environ $30 \%$ de la masse totale du matériau, la majorité de la masse étant attribuée aux nanotubes. Afin d'extraire des données expérimentales l'information concernant les $\mathrm{C}_{60}$, il est donc primordial de procéder par comparaison avec des données issues de nanotubes vides mesurées dans les mêmes conditions.

La densité d'état à basse température à été mesurée dans le domaine de fréquence totale [0,200 meV] en utilisant les spectromètres IN4C et IN1BeF. Les résultats sont montrés sur la figure 19 où la GDOS des peapods est directement comparée à celle des nanotubes et des $\mathrm{C}_{60}$. Dans le domaine des modes moléculaires du $\mathrm{C}_{60}(\mathrm{E}>30 \mathrm{meV})$ on voit clairement que celle-ci est issue de la somme pondérée des contributions des tubes et des fullerènes. En particulier dans les domaines où la densité d'état des SWNT est pauvre en contribution $(\mathrm{E}<50 \mathrm{meV}$ et $110 \mathrm{meV}<\mathrm{E}<140 \mathrm{meV}$ ) on reconnaît le profil des modes moléculaires du $\mathrm{C}_{60}$, le premier d'entre eux, le mode $\mathrm{H}_{g}^{1}$ "sortant" de la GDOS sous la forme d'un pic à $33 \mathrm{meV}$. Ceci suggère un couplage faible entre le tube et la chaîne des $\mathrm{C}_{60}$. L'évolution en température des premiers modes moléculaires du $\mathrm{C}_{60}$ est très intéressante (voir la figure 20). Elle fait apparaître la “disparition" progressive de leur contribution dès lors que la température est élevée au dessus de $100 \mathrm{~K}$. Cette disparition apparente peut se comprendre si l'on se rappelle que ces modes doivent être convolués par une fonction tenant compte des degrés de liberté de translation et de rotation de la molécule entière. De façon schématique, si l'on fait l'hypothèse que la rotation de la molécule est découplée de ses degrés de liberté interne (vibration atomique des atomes de carbone à la surface), la fonction de diffusion s'écrit sous la forme (voir le chapitre $1, \S 2.9$ p66 de la référence [38]) :

$$
\begin{aligned}
& S(\vec{Q}, \omega)=S^{R}(\vec{Q}, \omega) \otimes S^{v}(\vec{Q}, \omega) \\
& S(\vec{Q}, \omega)=S^{R}(\vec{Q}, \omega) \otimes\left[\left.S^{v}(\vec{Q})\right|_{e l} \delta(\omega)+\left.S^{v}(\vec{Q}, \omega)\right|_{\text {inel }}\right] \\
& S(\vec{Q}, \omega)=\left.S^{v}(\vec{Q})\right|_{e l} S^{R}(\vec{Q}, \omega)+\left.S^{R}(\vec{Q}, \omega) \otimes S^{v}(\vec{Q}, \omega)\right|_{\text {inel }}
\end{aligned}
$$




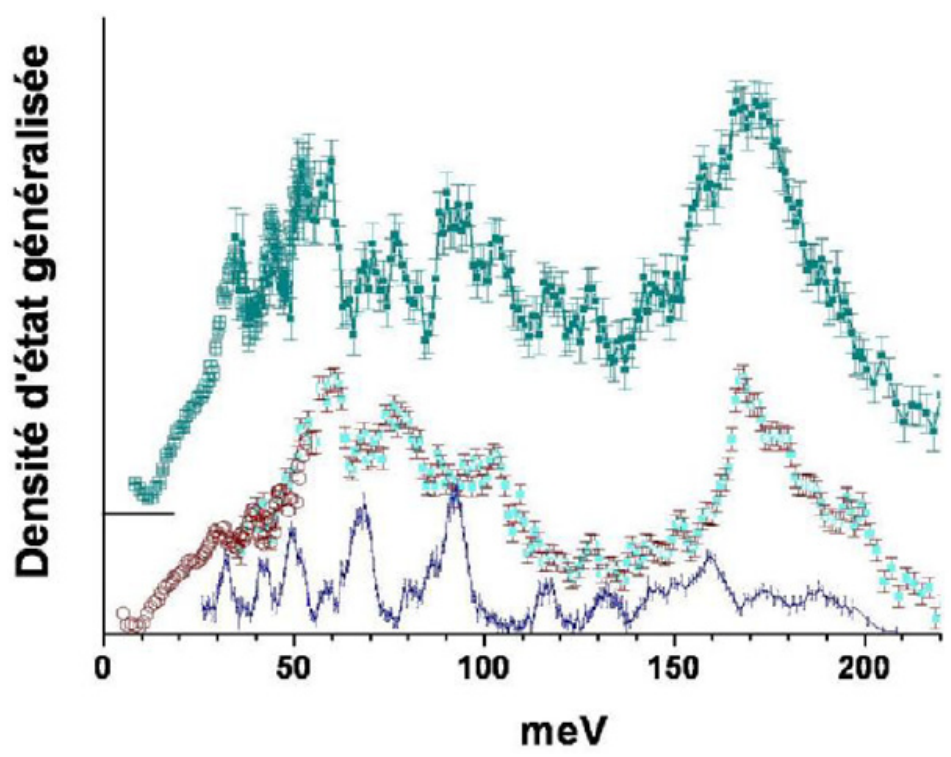

Figure 19. Densité d'état généralisée de peapods (courbe du haut), comparée à celle des nanotubes vides (courbe du milieu) et à celle des $C_{60}$ (courbe du bas). Les courbes sont issues de la combinaison des mesures effectuées sur les spectromètres IN1BeF (domaine d'énergie de 25 à $250 \mathrm{meV}$ ) et IN4C (domaine d'énergie de 6 à $55 \mathrm{meV}$ ), sauf pour le $\mathrm{C}_{60}$ où seul IN1BeF a été présenté sur cette figure.

où $S^{R}(\vec{Q}, \omega)$ et $S^{v}(\vec{Q}, \omega)$ sont les fonctions de diffusion associées aux mouvements de rotation d'ensemble de la molécule et aux vibrations moléculaires respectivement (les indices el et inel indiquent les parties élastiques et inélastiques de la fonction de diffusion). Dans le cas où la rotation prend le caractère temporel d'une relaxation, sa fonction de diffusion est la somme d'un certain nombre de lorentziennes, dont les largeurs sont caractéristiques des temps de relaxation. La fonction $\left.S^{v}(\vec{Q}, \omega)\right|_{\text {inel }}$ étant la somme de fonctions $\delta\left(\omega-\omega_{j}\right)$ (voir par exemple l'équation 9.57 du chapitre III), le spectre de la GDOS sera composé de la somme de fonctions lorentziennes centrées aux fréquences des modes moléculaires :

$$
\begin{aligned}
& G \operatorname{DOS}(\bar{Q}, \omega) \propto \sum_{j} S^{R}\left(\bar{Q}, \omega-\omega_{j}\right) \\
& G \operatorname{DOS}(\bar{Q}, \omega) \propto \sum_{j} \frac{\Gamma}{\left(\omega-\omega_{j}\right)^{2}+\Gamma^{2}}
\end{aligned}
$$

Si la relaxation est rapide, la largeur des lorentziennes $\Gamma$ sera d'autant plus grande, rendant les modes moléculaires très larges dans le spectre.

Dans les peapods, le désordre dynamique en question est lié aux rotations des molécules de $\mathrm{C}_{60}$. En effet, un signal quasiélastique est clairement observé dans les spectres, de largeur $\Gamma \sim 6$ meV à température ambiante. La dépendance de l'intensité de ce signal en fonction du vecteur de diffusion $\mathrm{Q}$ est représentée sur la figure 21. Cette forme est identique à celle du $\mathrm{C}_{60}$ représentée sur la figure 9.

Par contre, la dépendance en température du signal lié aux rotations des $\mathrm{C}_{60}$ est fortement différente lorsque les molécules sont confinées. En effet, aucun signal clair du gel de la rotation à basse température n'est observé. En particulier, aucun pic associé à la libration n'apparaît, même dans les spectres mesurés à des températures aussi basses que $2.5 \mathrm{~K}$. La dépendance de la susceptibilité mesurée à $\mathrm{Q}=3.4 \AA^{-1}$ fait apparaître un saut entre $100 \mathrm{~K}$ et $200 \mathrm{~K}$. Ceci est caractéristique de la perte de la composante 


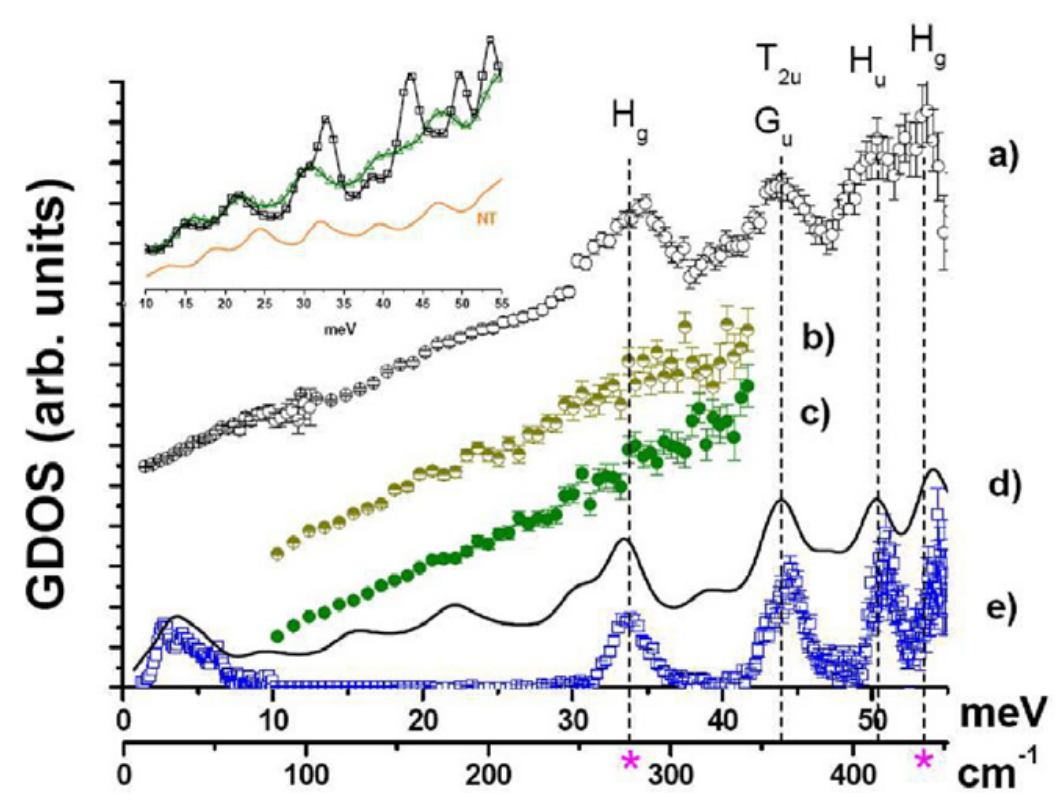

Figure 20. Densités d'état généralisées de peapods mesurées sur le spectromètre IN4C à $10 \mathrm{~K}$ (a), $150 \mathrm{~K}$ (b) et $300 \mathrm{~K}$ (c), comparées à celle (d) simulée à l'aide du modèle décrit dans la référence [37] (color online). La GDOS d'un échantillon de $\mathrm{C}_{60}$ mesuré dans les mêmes conditions et à $10 \mathrm{~K}$ est aussi montrée (e). Encart: Densité d'état calculée pour un peapod modèle (carrés et triangles) et pour un modèle de tube seul (ligne continue notée NT). Les courbes calculées ont été convoluées avec une fonction gaussienne (de largeur à mi-hauteur $=2 \mathrm{meV}$ ) pour tenir compte de la résolution du spectromètre dans ces conditions. La partie de la GDOS provenant des modes du $\mathrm{C}_{60} \mathrm{de}$ la courbe théorique faite de triangles a de plus été convoluée avec une Lorentzienne de largeur $6 \mathrm{meV}$ pour tenir compte de la diffusion rotationnelle des $\mathrm{C}_{60}$ (voir texte). Cette convolution a pour effet de diluer la contribution des modes moléculaires des fullerènes, et de perdre les pics fins associés à ces modes au profit d'une légère modulation du niveau de la GDOS des nanotubes. Cette "disparition" apparente permet de comprendre l'évolution de la GDOS en fonction de la température. D’après la référence [37]

quasiélastique dans ce domaine de température, et révèle un fort ralentissement de la dynamique de rotation, d'un caractère diffusif vers un caractère de type libration. Dans la fenêtre temporelle d'IN4, la diffusion rotationnelle est bloquée entre $100 \mathrm{~K}$ et $200 \mathrm{~K}$, soit au minimum $60 \mathrm{~K}$ au dessous de la transition "ordre-désordre" du $\mathrm{C}_{60}$. Cependant, la non-observation d'un pic lié aux librations suggère qu'aucune mise en ordre orientationnel n'apparaît dans ce système, même pour des températures aussi basses que $2.5 \mathrm{~K}$. On ne peut donc associer le ralentissement de la dynamique à une transition de premier ordre mais plutôt à un ralentissement progressif de la rotation des molécules de $\mathrm{C}_{60}$ confinées jusqu'à un "blocage" apparent dans la fenêtre temporelle d'IN4C. Les molécules effectuent alors des mouvements de réorientations à basse température, et librent à des fréquences dont la distribution est représentative de la surface de potentiel explorée par des molécules confinées orientées différemment.

Pour des nanotubes de diamètre proche de celui des tubes utilisés pour synthétiser ces peapods, il a été calculé que les barrières de potentiel séparant les orientations privilégiées d'un $\mathrm{C}_{60}$ unique confiné dans un tube sont très faibles [39]. Les contraintes exercées par le tube sur le fullerène inséré portent donc principalement sur la position du centre de masse de cette molécule, qui doit être sur l'axe longitudinal pour des tubes de diamètre $\leq 14 \AA$. Dès lors ce sont les interactions inter- $\mathrm{C}_{60}$ qui deviennent prépondérantes en ce qui concerne les orientations, et on se trouve dans un système qui peut être assimilé à un modèle d'Ising $1 \mathrm{D}$, pour lequel les $\mathrm{C}_{60}$ peuvent adopter statistiquement un grand nombre d'orientations et où aucune transition de phase "ordre-désordre" ne peut survenir à température finie. Nous renvoyons le lecteur intéressé par ce sujet aux références [37] et [40]. 


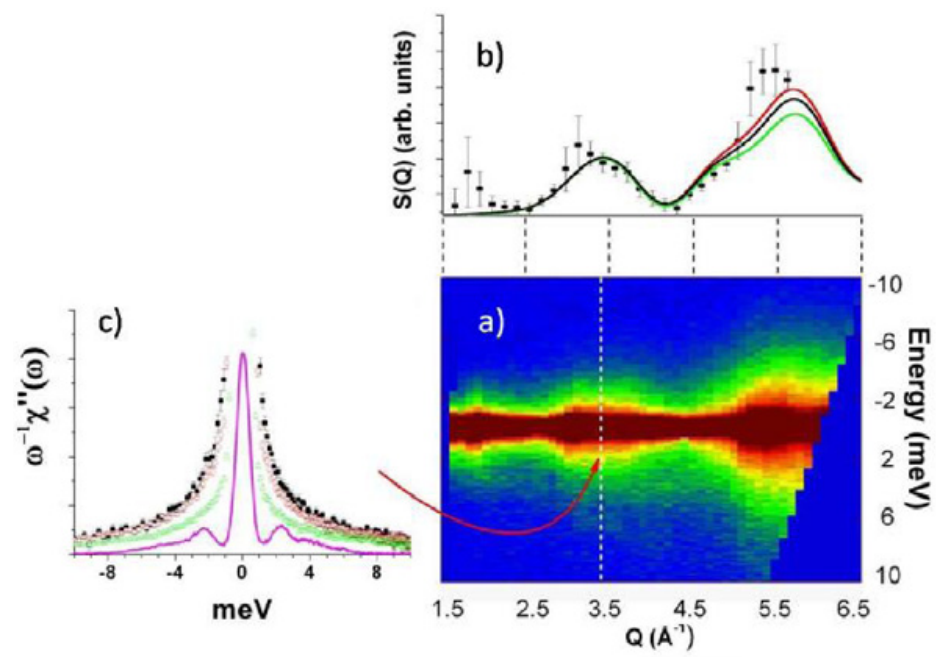

Figure 21. a) : Facteur de structure dynamique $S(Q, \omega)$ d'un échantillon de peapods à $280 \mathrm{~K}$. - b) : Evolution de l'intensité du signal QE en fonction de $\mathrm{Q}$. Le profil de cette évolution peut se fitter très convenablement avec un modèle de rotation isotrope, mais aussi avec un modèle de rotation uniaxiale. On peut donc ici seulement se limiter à l'affirmation que les $\mathrm{C}_{60}$ sont en rotation à $280 \mathrm{~K}$, sans pouvoir statuer sur le type de rotation. - c) : Evolution de la susceptibilité mesurée en fonction de la température à $\mathrm{Q}=3.4 \AA^{-1}$. La courbe en ligne continue rose représente la susceptibilité d'un échantillon de $\mathrm{C}_{60}$ pur à $200 \mathrm{~K}$. D'après la référence [37].

\section{CONCLUSION}

Au cours de ce chapitre, nous avons donné un aperçu de ce que la diffusion des neutrons a pu apporter à l'étude de la dynamique des composés originaux que représentent les fullerènes et les nanotubes de carbone. Nous avons décrit les principales caractéristiques des excitations intra et inter-moléculaires dans les nanotubes de carbone et dans les différentes phases que le $\mathrm{C}_{60}$ cristallin forme en fonction de la température. Nous avons ensuite présenté la modification des spectres et de la dynamique de ces systèmes lorsque des molécules de cubane sont intercalées dans les sites octaédriques du réseau du $\mathrm{C}_{60}$, ou encore quand les fullerènes sont confinés sur un réseau unidimensionel dans les nanotubes de carbone, formant les peapods. Nous avons vu que la transition "ordre-désordre" pouvait survenir à des températures différentes suivant l'environnement moléculaire du $\mathrm{C}_{60}$.

Devant l'impossibilité matérielle de fournir une revue exhaustive des résultats issus de diffusion neutronique sur les fullerènes, nous avons été contraints de réaliser des choix difficiles et de passer sous silence de très belles études sur les autres types de fullerènes $\left(\mathrm{C}_{70}\right)$, sur l'étude des fullerènes sous pression, sur les fullerènes dopés aux alcalins, etc. Pour les mêmes raisons, nous avons rarement évoqué les études faisant appel à d'autres techniques expérimentales (diffusion des rayons $\mathrm{X}$, spectroscopie Raman, $\mathrm{RMN}, .$. ) et qui ont apporté des résultats passionnants sur la dynamique des fullerènes et des nanotubes de carbone. Nous espérons du moins avoir suscité la curiosité des lecteurs et avoir donné à quelques-uns l'envie de se plonger dans ce joli sujet d'études.

\section{Remerciements}

Nous tenons à remercier nos collègues Robert Almairac, Pascale Launois et Jean-Louis Sauvajol pour leur investissement dans les études Raman, DRX et neutron des nanotubes de carbone, des peapods et des fullerènes. Nous remercions aussi Hiromishi Kataura et Sandor Pekker pour avoir eu la gentillesse de nous confier les échantillons de peapods et de fullerène-cubane préparés par leurs soins. Sacha Ivanov, Helmut Schober, Hannu Mutka, John Stride, Jean-Marc Zanotti, Jacques Ollivier et José Dianoux furent d'une aide considérable lors des 
expériences de diffusion inélastique des neutrons et lors de l'analyse des résultats de mesure. Nous voudrions aussi remercier Mark Johnson et Eric Pellegrini pour leur aide lors de la préparation des simulations numériques ainsi dans l'exploitation des trajectoires issues des simulations de dynamique moléculaire.

\section{Références}

[1] H.W. Kroto et al., Nature 318, 163 (1985)

[2] S. Iijima, Nature 354, 56 (1991)

[3] V.A. Davydov et al., Phys. Rev. B 61, 1936 (2000)

[4] J.R.D. Copley, Neutron News 4, 20 (1993)

[5] P. Launois, S. Ravy and R. Moret, Int. J. Mod. Phys. B 13, 253 (1999)

[6] R. Tycko et al., Phys. Rev. Lett. 67, 1886 (1991)

[7] R. Moret et al., J. Phys. I (France) 2, 1699 (1992)

[8] D.A. Neumann et al., Phys. Rev. Lett. 67, 3808 (1991)

[9] J. Yu et al., Appl. Phys. Lett. 63, 3152 (1993)

[10] R.L. Capelletti et al., Phys. Rev. Lett. 66, 3261 (1991)

[11] C. Coulombeau et al., J. Phys. Chem. 96, 22 (1992)

[12] K. Prassides et al., Chem. Phys. Lett. 187, 455 (1991)

[13] L. Pintschovius and S.L. Chaplot, Z. Phys. B 98, 527 (1995)

[14] L. Pintschovius et al., Phys. Rev. Lett. 69, 2662 (1992)

[15] L. Pintschovius et al., Rep. Prog. Phys. 59, 473 (1996)

[16] D. Lamoen and K.H. Michel, Phys. Rev. B 48, 807 (1993)

[17] B. Renker et al., Z. Phys. B 90, 325 (1993)

[18] H. Schober et al., Phys. Rev. B 59, 3287 (1999)

[19] W.I.F. David et al., Europhys. Lett. 18, 219 (1992)

[20] S. Pekker et al., Nature Mater. 4, 764 (2005)

[21] T. Yildirim et al., Chem. Phys. Lett. 309,234 (1999)

[22] C. Bousige et al., submitted to Phys. Rev. B (2010)

[23] N. M. Nemes, et al., J. Phys. Chem. B 113, 2042 (2008)

[24] A. Loiseau et al., Understanding Carbon Nanotubes, Lect. Notes Phys. 677, Springer (2006)

[25] L. Henrard et al., Eur. Phys. J. B 13, 661 (1999)

[26] L.-H. Ye et al., Phys. Rev. B 69, 235409 (2004)

[27] S. Rols et al., Eur. Phys. J. B 18, 201 (2000)

[28] S. Rols et al., Phys. Rev. Lett. 85, 5222 (2000)

[29] J.-L. Sauvajol, E. Anglaret, S. Rols et L. Alvarez, Carbon 40, 1697 (2002)

[30] J. C. Lasjaunias, et al. Phys. Rev. Lett. 91, 025901 (2003)

[31] J. C. Lasjaunias, C. R. Physique 4, 1047 (2003)

[32] B. W. Smith, M. Monthioux and D. E. Luzzi, Nature 396, 323 (1998)

[33] B. Burteau, et al., Chem. Phys. Lett. 310, 21 (1999)

[34] H. Kataura et al., Synthetic Metals 121, 1195 (2001)

[35] H. Kataura et al., Proceedings of the 25th International Conference on the Physics of Semiconductors, Osaka International Convention Center, Japan, September 2000

[36] J. Cambedouzou et al., Eur. J. B 42, 31 (2004)

[37] S. Rols et al., Phys. Rev. Lett. 101, 065507 (2008)

[38] M. Bée, Quasielastic Neutron Scattering: Principles and Applications in Solid State Chemistry, Biology and Material Science, Adam and Hilger, Bristol (1988)

[39] B. Verberck and K.H. Michel, Phys. Rev. B 74, 045421 (2006)

[40] K. Matsuda, Y. Maniwa and H. Kataura, Phys. Rev. B 77, 075421 (2008) 